\title{
Asymmetric Glycolate Aldol Reactions Using Cinchonium Phase-Transfer Catalysts
}

\author{
Merritt B. Andrus, * Jing Liu, Zhifeng Ye, John F. Cannon \\ Brigham Young University, Department of Chemistry and Biochemistry, \\ C100 BNSN, Provo, UT 84602-5700 \\ email:mbandrus@chem.byu.edu
}

Supporting Information

\begin{tabular}{|c|c|}
\hline Experimental & S-1-10 \\
\hline NMR Spectra & S-11-20 \\
\hline HPLC & S-21-28 \\
\hline X-ray Data & S-29-34 \\
\hline
\end{tabular}

\section{Experimental}

Air and water sensitive reactions were performed in flame-dried glassware under a nitrogen atmosphere. Water sensitive reagents were introduced via dry syringe or cannula. Methylene chloride, toluene, THF, diethyl ether, DMSO, DMF, methanol, triethylamine and pyridine were dried by passing through columns of activated alumina. Hexanes were distilled with $\mathrm{CaH}_{2}$. Reagents were purchased from Aldrich and Lancaster. Flash chromatography was carried out using 60-230 mesh silica gel. Radial chromatography was performed using 1, and 2 mm plates loaded with 230-400 mesh PF254 gypsum bound silica. Analytical thin-layer chromatography (TLC) was performed with Merck silica gel $60 \mathrm{~F}_{254}, 0.25 \mathrm{~mm}$ pre-coated TLC plates. TLC plates were visualized using $\mathrm{UV}_{254}$ and cerium molybdate with charring. All ${ }^{1} \mathrm{H}$ NMR spectra were obtained with either 300 or $500 \mathrm{MHz}$ Varian spectrometers using TMS (0.0 ppm) or chloroform (7.27 ppm) as an internal reference. Signals are reported as m (multiplet), s (singlet), d (doublet), t (triplet), q (quartet), bs (broad singlet), and dd (doublet doublet); and coupling constants are reported in hertz $(\mathrm{Hz}) .{ }^{13} \mathrm{C}$ NMR spectra were obtained with either 75 or $125 \mathrm{MHz}$ Varian spectrometers using chloroform (77.23 ppm) as the internal standard. Mass spectral data (HRMS, EI, FAB) were obtained from the Brigham Young University mass spectrometry facility. Optical rotations were obtained using the sodium $\mathrm{D}$ line at ambient temperature. Low temperatures were maintained using an immersion cooler with a cooling probe placed in an acetone bath.

\section{Substrate preparation}


<smiles>COc1ccc(OC)c(/C(=C/O[Na])O[Na])c1</smiles>

(Z)-(2-(Benzhydryloxy)-1-(2,5-dimethoxyphenyl)ethenyloxy)trimethyl silane (9). 2Benzhydryloxy-1-(2,5-dimethoxy-phenyl)-ethanone 1 (3 mmol, $1.09 \mathrm{~g}$ ) was dissolved in $10 \mathrm{ml}$ dry THF. LDA $(2.5 \mathrm{~mL})$ was drop-wisely added at $-78^{\circ} \mathrm{C}$. After 1 hour, TMSCl (2.5 mL) was added. The mixture was stirred for another 1 hour and the color was changed to pale yellow from orange. The reaction was warmed to room temperature. And THF was removed under reduced pressure. Dry hexanes were added. The white participates were removed by filtration. After removal of hexanes, the residue was recrystallized with hexanes and give 2 as white solid $(0.80 \mathrm{~g}, 61 \%)$. Data are: ${ }^{1} \mathrm{H}$ NMR $\left(\mathrm{CDCl}_{3}, 500 \mathrm{MHz}\right): \delta 0.17$ (s, $\left.9 \mathrm{H}\right), 3.61$ (s, $\left.3 \mathrm{H}\right), 3.75$ (s, $\left.3 \mathrm{H}\right), 5.79$ (s, $\left.1 \mathrm{H}\right), 6.63$ (dd, $J$ = 3.0, $9.0 \mathrm{~Hz}, 1 \mathrm{H}), 6.72$ (d, $J=9.0 \mathrm{~Hz}, 1 \mathrm{H}), 7.07$ (d, $J=0.3 \mathrm{~Hz}, 1 \mathrm{H}), 7.26-7.28$ (m, 2 $\mathrm{H}), 7.34$ (t, $J=7.5 \mathrm{~Hz}, 4 \mathrm{H}), 7.41$ (d, $\left.J=7.5 \mathrm{~Hz}, 4 \mathrm{H}) ;{ }^{13} \mathrm{C} \mathrm{NMR} \mathrm{(CDCl} 3,125 \mathrm{MHz}\right): \delta$ 0.8 , 55.8, 56.2, 85.7, 112.1, 112.91, 112.94, 126.9, 127.5, 127.9, 128.6, 131.0, 135.4, 141.5, 150.1, 153.8; HRMS (FAB ${ }^{+}$) found 457.1806, calcd 457.1811 for $\mathrm{C}_{26} \mathrm{H}_{30} \mathrm{O}_{4} \mathrm{SiNa}$.

\section{Bifluoride salt preparation}

Amberlyst A-26 ( $\mathrm{Br}^{-}$form, $\left.1.6 \mathrm{~g}, 9.6 \mathrm{meq}\right)$ column was flushed with $1 \mathrm{M}$ aqueous $\mathrm{NaOH}$ until all of the $\mathrm{Br}^{-}$was exchanged by $\mathrm{OH}^{-}$. The column was washed with $\mathrm{H}_{2} \mathrm{O}$ until it was neutral. Then it was flushed with $\mathrm{MeOH}$. Cinchonium bromide $(0.5 \mathrm{mmol})$ was dissolved in $10 \mathrm{~mL} \mathrm{MeOH}$ and slowly passed through the column. The column was eluted with $\mathrm{MeOH}$. The eluent was neutralized with $1 \mathrm{M} \mathrm{HF}(1 \mathrm{~mL})$. The solvents were removed under reduced pressure. Data for $4-\mathrm{CN}^{+} \mathrm{HF}_{2}^{-}$are: ${ }^{1} \mathrm{H}$ NMR $\left(\mathrm{CDCl}_{3}, 500 \mathrm{MHz}\right): \delta 0.94(\mathrm{t}$, $J=7 \mathrm{~Hz}, 3 \mathrm{H}), 1.11(\mathrm{~m}, 1 \mathrm{H}), 1.58(\mathrm{~m}, 2 \mathrm{H}), 1.76(\mathrm{~m}, 2 \mathrm{H}), 1.94(\mathrm{~m}, 1 \mathrm{H}), 2.01(\mathrm{~m}, 1 \mathrm{H})$, 2.31( t, $J=11.5 \mathrm{~Hz}, 1 \mathrm{H}), 2.88$ (q, $J=10 \mathrm{~Hz}, 1 \mathrm{H}), 3.22$ (t, $J=10.5 \mathrm{~Hz}, 1 \mathrm{H}), 4.00$ (m, 1H), $4.04(\mathrm{~m} .1 \mathrm{H}), 4.26(\mathrm{~m}, 1 \mathrm{H}), 4.49(\mathrm{~m}, 1 \mathrm{H}), 4.55(\mathrm{t}, J=11 \mathrm{~Hz}, 1 \mathrm{H}), 4.78(\mathrm{~d}, J=12.5 \mathrm{~Hz}$, 1H), 5.39 (d, $J=1 \mathrm{~Hz}, 1 \mathrm{H}), 5.42$ (d, $J=1.5 \mathrm{~Hz}, 1 \mathrm{H}), 5.82$ (d, $J=12 \mathrm{~Hz}, 1 \mathrm{H}), 6.07$ (m, 1H), 6.22 (bs, 1H), 7.21 (m, 1H), 7.61 (bs, 1H), 7.79 (t, $J=7.5 \mathrm{~Hz}, 1 \mathrm{H}), 7.90$ (bs, $1 \mathrm{H})$, $8.02(\mathrm{~m}, 1 \mathrm{H}), 8.15$ (d, $J=9 \mathrm{~Hz}, 1 \mathrm{H}), 8.59$ (d, $J=8 \mathrm{~Hz}), 8.98(\mathrm{~d}, J=4.5,1 \mathrm{H}) ;{ }^{13} \mathrm{C} \mathrm{NMR}$ $\left(\mathrm{CDCl}_{3}, 125 \mathrm{MHz}\right): \delta 11.5,22.1,24.3,24.5,24.7,36.2,54.6,56.7,57.5,66.8,70.5,70.7$, 74.2, 113.1, 113.9, 114.1, 119.1, 119.8, 120.3, 124.2, 125.4, 125.7, 128.4, 129.0, 129.2, 130.1, 130.4, 131.3, 132.4, 139.0, 139.1, 140.0, 141.0, 148.7, 149.7, 150.2, 150.3, 151.9, 152.2, 152.3, 153.9; HRMS $\left(\mathrm{FAB}^{+}\right.$) found 504.2364, calcd 504.2359 for $\mathrm{C}_{29} \mathrm{H}_{32} \mathrm{~F}_{3} \mathrm{~N}_{2} \mathrm{NaO}$.

\section{General procedure of the asymmetric aldol reaction}<smiles>COc1ccc(OC)c(C(=O)C(O[Na])[C@H](O)c2ccccc2)c1</smiles> 
2-(Benzhydryloxy)-1-(2,5-dimethoxyphenyl)-3-hydroxy-3-phenylpropan-1-one (8, $\mathbf{R}=\mathbf{P h}$ ). O(9)-allyl-N-2',3',4'-triflurorbenzylhydrocinchonium bifluoride salt $\mathbf{4}-\mathbf{C N}^{+} \mathbf{H F}_{2}{ }^{-}$ (15 mg, $0.003 \mathrm{mmol})$ was dissolved in reagent $1.5 \mathrm{~mL}$ THF. Benzaldehyde $(0.3 \mathrm{mmol}$, $0.0318 \mathrm{~g}$ ) was added and the mixture was cooled to $-55^{\circ} \mathrm{C}$. (Z)-(2-(benzhydryloxy)-1-(2,5dimethoxyphenyl)ethenyloxy)trimethyl silane (0.15 mmol, 0.0652g) 9 was added. After 24 hours, the reaction was quenched with $\mathrm{CsF}$ and $\mathrm{H}_{2} \mathrm{O}$. The solution was extracted 5 times by $\mathrm{CH}_{2} \mathrm{Cl}_{2}$. The combined organic layers were washed with water and brine, then dried over $\mathrm{MgSO}_{4}$, filtered and concentrated in vacuo. Radial chromatography afforded the desired compound 8 (0.054 g, 76\%). Its enatioselectivity can be enriched by recrystallization with $\mathrm{EtOH}$ and hexanes. The crystals were racemic product and the residue was the enatio-enriched product (65\%, 95\% ee). Data are: ${ }^{1} \mathrm{H}$ NMR $\left(\mathrm{CDCl}_{3}, 500\right.$ MHz): $\delta 3.13$ (b, $1 \mathrm{H}$ ), 3.55 (s, $3 \mathrm{H}$ ), 3.77 (s, $3 \mathrm{H}$ ), 5.03 (s, $1 \mathrm{H}$ ), 5.28 (d, $J=2.5 \mathrm{~Hz}, 1 \mathrm{H}$ ), 5.48 (s, $1 \mathrm{H}), 6.78-6.85$ (m, $3 \mathrm{H}), 6.98-7.00$ (m, $1 \mathrm{H}), 7.10-7.31$ (m, $14 \mathrm{H}) ;{ }^{13} \mathrm{C} \mathrm{NMR}$ $\left(\mathrm{CDCl}_{3}, 125 \mathrm{MHz}\right): \delta$ 55.97, 55.99, 73.9, 82.5, 84.7, 113.3, 114.3, 121.1, 126.2, 127.1, 127.3, 127.5, 127.6, 127.7, 127.8, 128.2, 128.3, 128.4, 140.5, 141.2, 142.2, 152.5, 154.0, 200.1; HRMS (FAB ${ }^{+}$) found 491.1844, calcd 491.1834 for $\mathrm{C}_{30} \mathrm{H}_{28} \mathrm{O}_{5} \mathrm{Na}$; HPLC (DAICEL Chiralpack AD, $10 \% \mathrm{EtOH} /$ hexane, flow rate $1.0 \mathrm{~mL} / \mathrm{min}): \mathrm{t}_{\mathrm{R}}=23.1$ (minor). $\mathrm{t}_{\mathrm{R}}=33.0$ (major).<smiles>COc1ccc([C@@H](O)[C@H](O[Na])C(=O)c2cc(OC)ccc2OC)cc1</smiles>

2-(Benzhydryloxy)-1-(2,5-dimethoxyphenyl)-3-hydroxy-3-(4-methoxyphenyl)

propan-1-one. $8(\mathbf{R}=4-\mathbf{M e O P h})$ Data are: ${ }^{1} \mathrm{H}$ NMR $\left(\mathrm{CDCl}_{3}, 500 \mathrm{MHz}\right): \delta 3.06(\mathrm{~d}, J=$ $7.5 \mathrm{~Hz}, 1 \mathrm{H}$ ), 3.54 (s, $3 \mathrm{H}$ ), 3.76 (s, $3 \mathrm{H}), 3.81$ (s, $3 \mathrm{H}), 4.97$ (dd, $J=3.5,7.5 \mathrm{~Hz}, 1 \mathrm{H}$ ), $5.26(\mathrm{~d}, J=3.5 \mathrm{~Hz}, 1 \mathrm{H}), 6.76-6.81(\mathrm{~m}, 3 \mathrm{H}), 6.93-6.99(\mathrm{~m}, 3 \mathrm{H}), 7.14-7.33(\mathrm{~m}, 11 \mathrm{H})$; ${ }^{13} \mathrm{C} \mathrm{NMR}\left(\mathrm{CDCl}_{3}, 125 \mathrm{MHz}\right): \delta 55.5,56.0,73.8,82.6,84.9,113.3,113.6,114.3,120.9$, 127.4, 127.6, 127.8, 128.0, 128.3, 128.5, 128.7, 133.1, 140.7, 142.3, 152.5, 154.0, 159.2, 200.4; HRMS $\left(\mathrm{FAB}^{+}\right.$) found 521.1940, calcd 521.1940 for $\mathrm{C}_{31} \mathrm{H}_{30} \mathrm{O}_{6} \mathrm{Na}$; HPLC (DAICEL Chiralpack AD, $10 \% \mathrm{EtOH} /$ hexane, flow rate $1.0 \mathrm{~mL} / \mathrm{min}): \mathrm{t}_{\mathrm{R}}=36.1$ (minor). $\mathrm{t}_{\mathrm{R}}=49.8$ (major).<smiles>COc1ccc(OC)c(C(=O)C(O[Na])[C@H](O)c2ccc(C)cc2)c1</smiles>

2-(Benzhydryloxy)-1-(2,5-dimethoxyphenyl)-3-hydroxy-3-p-tolylpropan-1-one. 8 (R=4-MePH) Data are: ${ }^{1} \mathrm{H}$ NMR $\left(\mathrm{CDCl}_{3}, 500 \mathrm{MHz}\right): \delta 2.35$ (s, $\left.3 \mathrm{H}\right), 3.05$ (d, $J=7.5 \mathrm{~Hz}$, $1 \mathrm{H}), 3.54$ (s, $3 \mathrm{H}$ ), 3.76 (s, $3 \mathrm{H}$ ), 4.99 (dd, $J=3.0,7.5 \mathrm{~Hz}, 1 \mathrm{H}), 5.26$ (d, $J=3.0 \mathrm{~Hz}, 1 \mathrm{H}$ ), 5.48 (s, 1 H), 6.78 (d, $J=9$ Hz, 1 H), 6.88-6.90 (m, 2 H), 6.96-6.99 (m, 1 H), 7.07-7.32 (m, $13 \mathrm{H}) ;{ }^{13} \mathrm{C} \mathrm{NMR}\left(\mathrm{CDCl}_{3}, 125 \mathrm{MHz}\right): \delta$ 21.3, 56.0, 73.9, 82.6, 84.9, 113.3, 114.3, 
120.9, 126.2, 127.3, 127.5, 127.7, 127.9, 128.3, 128.4, 128.7, 128.9, 137.1, 138.1, 140.7, 142.3, 152.5, 154.0, 200.3; HRMS $\left(\mathrm{M}^{+}\right)$found 482.1078, calcd 482.2093 for $\mathrm{C}_{31} \mathrm{H}_{30} \mathrm{O}_{5}$; HPLC (DAICEL Chiralpack AD, 10\% EtOH/hexane, flow rate $1.0 \mathrm{~mL} / \mathrm{min}$ ): $\mathrm{t}_{\mathrm{R}}=21.0$ (minor). $t_{R}=32.3$ (major).<smiles>COc1ccc(OC)c(C(=O)C(O)[C@H](O)c2ccc(Cl)cc2)c1</smiles>

2-(Benzhydryloxy)-3-(4-chlorophenyl)-1-(2,5-dimethoxyphenyl)-3-hydroxypropan1-one. 8 (R=p-ClPh) Data are: major: ${ }^{1} \mathrm{H}$ NMR $\left(\mathrm{CDCl}_{3}, 500 \mathrm{MHz}\right): \delta 3.20$ (bs, $\left.1 \mathrm{H}\right)$, 3.56 (s, 3 H), 3.77 (s, 3 H), 4.99 (s, 1 H), 5.22 (dd, $J=1.0$ Hz), 5.47 (s, 1 H), 6.79-6.86 (m, $3 \mathrm{H}), 7.00-7.02$ (m, $1 \mathrm{H}), 7.13-7.32$ (m, $13 \mathrm{H}) ;{ }^{13} \mathrm{C} \mathrm{NMR}\left(\mathrm{CDCl}_{3}, 125 \mathrm{MHz}\right): \delta 55.9$, 56.0, 73.3, 82.6, 84.5, 113.4, 114.3, 121.1, 127.2, 127.6, 127.9, 128.2, 128.3, 128.4, 133.1, 139.8, 140.2, 142.0, 152.5, 154.0, 199.8; HRMS $\left(\mathrm{FAB}^{+}\right)$found 525.1457, calcd 525.1445 for $\mathrm{C}_{30} \mathrm{H}_{27} \mathrm{O}_{5} \mathrm{ClNa}$; HPLC (DAICEL Chiralpack AD, 10\% EtOH/hexane, flow rate 1.0 $\mathrm{mL} / \mathrm{min}$ ): $\mathrm{t}_{\mathrm{R}}=23.2$ (minor). $\mathrm{t}_{\mathrm{R}}=28.9$ (major).<smiles></smiles>

2-(Benzhydryloxy)-3-(biphenyl-4-yl)-1-(2,5-dimethoxyphenyl)-3-hydroxypropan-1one. 8 (4-biphenyl) Data are: ${ }^{1} \mathrm{H}$ NMR $\left(\mathrm{CDCl}_{3}, 500 \mathrm{MHz}\right): \delta 3.14(\mathrm{~d}, J=8.0 \mathrm{~Hz}, 1 \mathrm{H})$, 3.60 (s, $3 \mathrm{H}$ ), 3.77 (s, $3 \mathrm{H}$ ), 5.07 (dd, $J=3.0,8.0 \mathrm{~Hz}, 1 \mathrm{H}$ ), 5.31 (d, $J=3.0 \mathrm{~Hz}, 1 \mathrm{H}$ ), 5.50 (s, $1 \mathrm{H}), 6.80$ (d, $J=9.0 \mathrm{~Hz}, 1 \mathrm{H}), 6.88-6.90(\mathrm{~m}, 2 \mathrm{H}), 7.00$ (dd, $J=3.0,9.0 \mathrm{~Hz}, 1 \mathrm{H}$ ), 7.10-7.38 (m, $12 \mathrm{H}$ ), 7.45-7.52 (m, $4 \mathrm{H}$ ), 7.60 (d, $J=8.0 \mathrm{~Hz}, 2 \mathrm{H}$ ); ${ }^{13} \mathrm{C} \mathrm{NMR}\left(\mathrm{CDCl}_{3}, 125\right.$ MHz): $\delta$ 56.0, 56.1, 73.8, 82.6, 84.9, 113.3, 114.4, 121.0, 126.7, 126.9, 127.1, 127.27, 127.32, 127.5, 127.6, 127.8, 127.9, 128.36, 128.43, 129.0, 140.2, 140.5, 141.2, 142.2, 152.6, 154.0, 200.2; HRMS $\left(\mathrm{FAB}^{+}\right.$) found 567.2141, calcd 567.2147 for $\mathrm{C}_{36} \mathrm{H}_{32} \mathrm{O}_{5} \mathrm{Na}$; HPLC (DAICEL Chiralpack AD, 10\% EtOH/hexane, flow rate $1.0 \mathrm{~mL} / \mathrm{min}$ ): $\mathrm{t}_{\mathrm{R}}=33.6$ (minor). $t_{R}=38.2$ (major).<smiles>COc1ccc(OC)c(C(=O)[C@H](O)[C@H](O)c2ccccc2OC)c1</smiles>

2-(Benzhydryloxy)-1-(2,5-dimethoxyphenyl)-3-hydroxy-3-(2-methoxyphenyl) propan-1-one. $8\left(\mathbf{R}=\boldsymbol{p}\right.$-CIPh) Data are: ${ }^{1} \mathrm{H}$ NMR $\left(\mathrm{CDCl}_{3}, 500 \mathrm{MHz}\right): \delta 3.23(\mathrm{~d}, J=9.0$ Hz, 1 H), 3.465 (s, 1 H), 3.47 (s, 1 H), 3.74 (s, 1 H), 5.19 (dd, $J=2.0,9.0$ Hz, 1 H), 5.35 
(d, $J=2.0 \mathrm{~Hz}, 1 \mathrm{H}$ ), 5.45 (s, $1 \mathrm{H}), 6.68-6.81$ (m, $4 \mathrm{H}), 6.92-7.02$ (m, $3 \mathrm{H}), 7.09-7.31$ (m, $9 \mathrm{H}), 7.43-7.45$ (m, $1 \mathrm{H}) ;{ }^{13} \mathrm{C}$ NMR $\left(\mathrm{CDCl}_{3}, 125 \mathrm{MHz}\right): \delta 54.9,55.7,55.9,70.2$, 82.3, 82.8, 110.0, 112.6, 114.2, 119.3, 120.5, 127.2, 127.4, 127.5, 127.7, 128.2, 128.3, 128.4, 128.5, 129.0, 141.0, 142.4, 152.0, 153.7, 156.0, 202.3; HRMS $\left(\mathrm{FAB}^{+}\right)$found 521.1954, calcd 521.1940for $\mathrm{C}_{31} \mathrm{H}_{30} \mathrm{O}_{6} \mathrm{Na}$; HPLC (DAICEL Chiralpack AD, 10\% EtOH/hexane, flow rate $1.0 \mathrm{~mL} / \mathrm{min}$ ): $\mathrm{t}_{\mathrm{R}}=19.0$ (minor). $\mathrm{t}_{\mathrm{R}}=31.8$ (major).<smiles>COc1ccc(OC)c(C(=O)[C@H](O)[C@H](O)c2ccccc2C)c1</smiles>

2-(Benzhydryloxy)-1-(2,5-dimethoxyphenyl)-3-hydroxy-3-o-tolylpropan-1-one, 8 (R=o-MePh) Data are: ${ }^{1} \mathrm{H}$ NMR $\left(\mathrm{CDCl}_{3}, 500 \mathrm{MHz}\right): \delta 1.94$ (s, $\left.3 \mathrm{H}\right), 3.07(\mathrm{~d}, J=7.0 \mathrm{~Hz}$, $1 \mathrm{H}), 3.41$ (s, $3 \mathrm{H}$ ), 3.75 (s, $3 \mathrm{H}$ ), 5.12 (dd, $J=3.5,7.0 \mathrm{~Hz}, 1 \mathrm{H}$ ), 5.28 (d, $J=3.5 \mathrm{~Hz}, 1 \mathrm{H}$ ), 5.47 (s, $1 \mathrm{H}), 6.71$ (d, $J=9.5 \mathrm{~Hz}, 1 \mathrm{H}), 6.84(\mathrm{~m}, 2 \mathrm{H}), 6.93(\mathrm{~m}, 1 \mathrm{H}), 7.00(\mathrm{~m}, 2 \mathrm{H}), 7.12-$ $7.32(\mathrm{~m}, 10 \mathrm{H}), 7.51(\mathrm{~m}, 1 \mathrm{H}) ;{ }^{13} \mathrm{C} \mathrm{NMR}\left(\mathrm{CDCl}_{3}, 125 \mathrm{MHz}\right): \delta$ 18.6, 55.5, 56.0, 71.3, 82.6, 82.7, 112.5, 114.1, 120.0, 125.8, 127.2, 127.3, 127.57, 127.62, 127.65, 127.73, 128.1, 128.35, 128.44, 130.3, 134.3, 138.3, 140.8, 142.2, 151.8, 153.8, 202.0; HRMS $\left(\mathrm{FAB}^{+}\right.$) found 505.1986, calcd 505.1991 for $\mathrm{C}_{31} \mathrm{H}_{30} \mathrm{O}_{5} \mathrm{Na}$; HPLC (DAICEL Chiralpack $\mathrm{AD}, 10 \% \mathrm{EtOH} /$ hexane, flow rate $1.0 \mathrm{~mL} / \mathrm{min}$ ): $\mathrm{t}_{\mathrm{R}}=12.2$ (minor). $\mathrm{t}_{\mathrm{R}}=21.3$ (major).<smiles>COc1ccc(OC)c(C(=O)[C@H](O)[C@H](O)[C@H](O)c2ccccc2-c2ccccc2)c1</smiles>

2-(Benzhydryloxy)-3-(biphenyl-2-yl)-1-(2,5-dimethoxyphenyl)-3-hydroxypropan-1one. 8 (R=2-biphenyl) Data are: major: ${ }^{1} \mathrm{H} \mathrm{NMR}\left(\mathrm{CDCl}_{3}, 500 \mathrm{MHz}\right): \delta 3.06(\mathrm{~d}, \mathrm{~J}=7.5 \mathrm{~Hz}$, $1 \mathrm{H}$ ), 3.29 (s, $3 \mathrm{H}$ ), 3.71 (s, $3 \mathrm{H}$ ), 4.76 (d, $J=2.5 \mathrm{~Hz}, 1 \mathrm{H}), 5.26$ (dd, $J=2.5,7.5 \mathrm{~Hz}, 1 \mathrm{H}$ ), 5.38 (s, $1 \mathrm{H}$ ), 6.50 (d, $J=9 \mathrm{~Hz}, 1 \mathrm{H}), 6.64$ (d, $J=3.0 \mathrm{~Hz}, 1 \mathrm{H}), 6.74-6.78$ (m, $4 \mathrm{H}), 6.80-$ $6.82(\mathrm{~m}, 1 \mathrm{H}), 7.02-7.42(\mathrm{~m}, 14 \mathrm{H}), 7.73(\mathrm{~d}, J=8.0 \mathrm{~Hz}, 1 \mathrm{H}) ;{ }^{13} \mathrm{C}$ NMR $\left(\mathrm{CDCl}_{3}, 125\right.$ MHz): $\delta$ 55.5, 56.0, 70.4, 82.4, 83.1, 112.3, 113.2, 118.5, 126.9, 127.3, 127.49, 127.55, 127.68, 127.71, 127.8, 128.1, 128.2, 128.3, 128.5, 128.9, 130.3, 137.7, 140.37, 140.44, 140.9, 142.3, 151.0, 153.5, 202.9; HRMS $\left(\mathrm{FAB}^{+}\right.$) found 567.2161, calcd 567.2147 for $\mathrm{C}_{36} \mathrm{H}_{32} \mathrm{O}_{5} \mathrm{Na}$; HPLC (DAICEL Chiralpack AD, $10 \% \mathrm{EtOH} /$ hexane, flow rate 1.0 $\mathrm{mL} / \mathrm{min}$ ): $\mathrm{t}_{\mathrm{R}}=16.8$ (minor). $\mathrm{t}_{\mathrm{R}}=21.0$ (major). 
<smiles>COc1ccc(OC)c(C(=O)C(O)C(O)c2ccccc2-c2ccccc2)c1</smiles>

minor ${ }^{1} \mathrm{H} \mathrm{NMR}\left(\mathrm{CDCl}_{3}, 500 \mathrm{MHz}\right): \delta 3.14$ (d, $\left.J=8.5 \mathrm{~Hz}, 1 \mathrm{H}\right), 3.55$ (s, $\left.3 \mathrm{H}\right), 3.75(\mathrm{~s}, 3 \mathrm{H})$, 5.09 (dd, $J=3.0,8.5 \mathrm{~Hz}, 1 \mathrm{H}), 5.34$ (d, $J=3.0 \mathrm{~Hz}, 1 \mathrm{H}), 5.51$ (s, $1 \mathrm{H}), 6.78$ (d, $J=9.0 \mathrm{~Hz}$, $1 \mathrm{H}$ ), 6.86-6.89 (m, $2 \mathrm{H}$ ), 6.97-7.00 (dd, $J=3.5,9.0 \mathrm{~Hz}, 1 \mathrm{H}$ ), 7.07-7.49 (m, $18 \mathrm{H})$; HRMS $\left(\mathrm{FAB}^{+}\right.$) found 567.2158, calcd 567.2147 for $\mathrm{C}_{36} \mathrm{H}_{32} \mathrm{O}_{5} \mathrm{Na}$; HPLC (DAICEL Chiralpack AD, $10 \% \mathrm{EtOH} /$ hexane, flow rate $1.0 \mathrm{~mL} / \mathrm{min}$ ): $\mathrm{t}_{\mathrm{R}}=33.8$ (major). $\mathrm{t}_{\mathrm{R}}=44.3$ (minor).<smiles>COc1ccc(OC)c(C(=O)[C@H](O[Na])[C@H](O)/C=C/c2ccccc2)c1</smiles>

(E)-2-(Benzhydryloxy)-1-(2,5-dimethoxyphenyl)-3-hydroxy-5-phenylpent-4-en-1-one. 8 (R=2-styryl) Data are: major ${ }^{1} \mathrm{H} \mathrm{NMR}\left(\mathrm{CDCl}_{3}, 500 \mathrm{MHz}\right): \delta 2.73$ (d, $\left.J=8.5 \mathrm{~Hz}, 1 \mathrm{H}\right)$, 3.56 (s, $3 \mathrm{H}$ ), 3.72 (s, $3 \mathrm{H}$ ), 4.56 (bs, $1 \mathrm{H}$ ), 5.12 (d, $J=3.0 \mathrm{~Hz}, 1 \mathrm{H}$ ), 5.62 (s, $1 \mathrm{H}), 6.13$ (dd, $J=5.5,16 \mathrm{~Hz}, 1 \mathrm{H}), 6.56$ (d, $J=16 \mathrm{~Hz}, 1 \mathrm{H}), 6.79$ (d, $J=9.5 \mathrm{~Hz}, 1 \mathrm{H}), 6.98$ (dd, $J=$ 3.0, $8.5 \mathrm{~Hz}, 1 \mathrm{H}), 7.15-7.41(\mathrm{~m}, 16 \mathrm{H}) ;{ }^{13} \mathrm{C} \mathrm{NMR}\left(\mathrm{CDCl}_{3}, 125 \mathrm{MHz}\right): \delta$ 55.96, 56.05, 73.0, 82.8, 84.1, 113.3, 114.2, 121.0, 126.7, 127.2, 127.5, 127.6, 127.8, 128.2, 128.3, 128.4, 128.6, 128.7, 129.4, 131.2, 136.8, 140.8, 142.1, 152.6, 154.1, 200.0; HRMS $\left(\mathrm{M}^{+}\right)$ found 494.2091, calcd 494.2093 for $\mathrm{C}_{32} \mathrm{H}_{30} \mathrm{O}_{5}$; HPLC (DAICEL Chiralpack AD, 10\% IPA/hexane, flow rate $1.0 \mathrm{~mL} / \mathrm{min}$ ): $\mathrm{t}_{\mathrm{R}}=30.8$ (minor). $\mathrm{t}_{\mathrm{R}}=35.7$ (major).<smiles>COc1ccc(OC)c(C(=O)[C@H](O[Na])[C@H](O)CCc2ccccc2)c1</smiles>

2-(Benzhydryloxy)-1-(2,5-dimethoxyphenyl)-3-hydroxy-5-phenylpentan-1-one. 8 (R=2-PhEt) Data are: major ${ }^{1} \mathrm{H}$ NMR $\left(\mathrm{CDCl}_{3}, 500 \mathrm{MHz}\right): \delta 1.78(\mathrm{~m}, 1 \mathrm{H}), 2.00(\mathrm{~m}, 1 \mathrm{H})$, 2.35 (d, $J=10 \mathrm{~Hz}, 1 \mathrm{H}), 2.44(\mathrm{~m}, 1 \mathrm{H}), 2.63$ (m, $1 \mathrm{H}), 3.46$ (s, $3 \mathrm{H}), 3.76$ (s, $3 \mathrm{H}), 3.84$ (dd, $J=1.5,10 \mathrm{~Hz}, 1 \mathrm{H}), 5.01$ (d, $J=1.5 \mathrm{~Hz}, 1 \mathrm{H}), 5.64$ (s, $1 \mathrm{H}), 6.76$ (d, $J=9.5 \mathrm{~Hz}, 1 \mathrm{H}$ ), 6.99 (dd, $J=3.5,8.5 \mathrm{~Hz}, 1 \mathrm{H}$ ), 7.07 (d, $J=7.0 \mathrm{~Hz}, 2 \mathrm{H}), 7.15-7.36$ (m, $12 \mathrm{H}$ ), 7.43 (d, $J=$ $7.5 \mathrm{~Hz}, 2 \mathrm{H}) ;{ }^{13} \mathrm{C} \mathrm{NMR}\left(\mathrm{CDCl}_{3}, 125 \mathrm{MHz}\right): \delta$ 32.0, 36.5, 55.8, 56.0, 71.6, 82.2, 83.0, 113.2, 114.2, 120.9, 126.0, 127.2, 127.3, 127.6, 128.27, 128.30, 128.5, 128.57, 128.60, 128.7, 141.0, 142.0, 142.3, 152.5, 154.0, 201.6; HRMS $\left(\mathrm{FAB}^{+}\right)$found 519.2149, calcd 519.2147 for $\mathrm{C}_{32} \mathrm{H}_{32} \mathrm{O}_{5} \mathrm{Na}$; HPLC (DAICEL Chiralpack AD, 10\% EtOH/hexane, flow rate $1.0 \mathrm{~mL} / \mathrm{min}$ ): $\mathrm{t}_{\mathrm{R}}=38.0$ (minor). $\mathrm{t}_{\mathrm{R}}=51.0$ (major). 
<smiles>COc1ccc(OC)c(C(=O)C(O[Na])[C@H](O)c2ccco2)c1</smiles>

2-(Benzhydryloxy)-1-(2,5-dimethoxyphenyl)-3-(furan-2-yl)-3-hydroxypropan-1-one. 8 (R=2-furyl) Data are: major: ${ }^{1} \mathrm{H}$ NMR $\left(\mathrm{CDCl}_{3}, 500 \mathrm{MHz}\right): \delta 3.09$ (d, $\left.J=9.0 \mathrm{~Hz}, 1 \mathrm{H}\right)$, 3.62 (s, 3 H), 3.78 (s, 3 H), 5.04 (dd, $J=2.5,9.0 \mathrm{~Hz}, 1 \mathrm{H}$ ), 5.42 (d, $J=2.5$ Hz, 1 H), 5.53 (s, 1 H), 6.29-6.35 (m, 2 H), 6.81 (d, $J=8.5$ Hz, 1 H), 7.00-7.05 (m, 3 H), 7.21-7.37 (m, $10 \mathrm{H}) ;{ }^{13} \mathrm{C} \mathrm{NMR}\left(\mathrm{CDCl}_{3}, 125 \mathrm{MHz}\right): \delta 55.9,56.0,69.2,82.6,82.7,107.2,110.6,113.3$, 114.1, 121.5, 126.3, 127.4, 127.7, 127.9, 128.3, 128.5, 140.6, 141.8, 142.1, 153.0, 153.9, 154.1, 198.9; HRMS (FAB ${ }^{+}$) found 481.1637, calcd 481.1627 for $\mathrm{C}_{28} \mathrm{H}_{26} \mathrm{O}_{6} \mathrm{Na}$; HPLC (DAICEL Chiralpack AD, 10\% EtOH/hexane, flow rate $1.0 \mathrm{~mL} / \mathrm{min}$ ): $\mathrm{t}_{\mathrm{R}}=21.4$ (minor). $\mathrm{t}_{\mathrm{R}}=38.3$ (major).<smiles>COc1ccc(OC)c(C(=O)C(O[Na])C(O)c2ccco2)c1</smiles>

minor: ${ }^{1} \mathrm{H}$ NMR $\left(\mathrm{CDCl}_{3}, 500 \mathrm{MHz}\right): \delta 2.94$ (d, $\left.J=8.5 \mathrm{~Hz}, 1 \mathrm{H}\right), 3.58$ (s, $\left.3 \mathrm{H}\right), 3.73$ (s, 3 H), 5.04 (dd, $J=5.5,8.5 \mathrm{~Hz}, 1 \mathrm{H}), 5.43$ (d, $J=5.5 \mathrm{~Hz}, 1 \mathrm{H}), 5.47$ (s, $1 \mathrm{H}), 6.28(\mathrm{~s}, 1 \mathrm{H})$, $6.32(\mathrm{~s}, 1 \mathrm{H}), 6.76(\mathrm{~d}, J=9.5 \mathrm{~Hz}, 1 \mathrm{H}), 6.95-6.99(\mathrm{~m}, 2 \mathrm{H}), 7.17-7.30(\mathrm{~m}, 11 \mathrm{H}) ;{ }^{13} \mathrm{C}$ NMR $\left(\mathrm{CDCl}_{3}, 125 \mathrm{MHz}\right): \delta$ 56.0, 56.2, 69.1, 82.0, 82.9, 108.5, 110.6, 113.4, 113.8, 120.8, 127.4, 127.6, 127.96, 128.0, 128.1, 128.3, 128.6, 141.0, 142.0, 142.04, 152.5, 153.2, 153.9, 200.6; HRMS (FAB ${ }^{+}$) found 481.1624, calcd 481.1627 for $\mathrm{C}_{28} \mathrm{H}_{26} \mathrm{O}_{6} \mathrm{Na}$; HPLC (DAICEL Chiralpack AD, 10\% EtOH/hexane, flow rate $1.0 \mathrm{~mL} / \mathrm{min}$ ): $\mathrm{t}_{\mathrm{R}}=27.5$ (minor). $\mathrm{t}_{\mathrm{R}}=31.0$ (major).<smiles>COc1ccc(OC)c(C(=O)C(O)[C@H](O)c2ccoc2)c1</smiles>

2-(Benzhydryloxy)-1-(2,5-dimethoxyphenyl)-3-(furan-3-yl)-3-hydroxypropan-1-one. 8 (R=3-furyl) Data are: ${ }^{1} \mathrm{H}$ NMR $\left(\mathrm{CDCl}_{3}, 500 \mathrm{MHz}\right): \delta 2.95$ (d, $\left.J=8.5 \mathrm{~Hz}, 1 \mathrm{H}\right), 3.56$ (s, 3 H), 3.77 (s, 3 H), 4.96 (dd, $J=2.5,8.5$ Hz, 1 H), 5.23 (d, $J=2.5$ Hz, 1 H), 5.60 (s, 1 H), 6.21 (s,1 H), 6.79 (d, $J=9.5 \mathrm{~Hz}, 1 \mathrm{H}), 7.00$ (q, $J=2.5 \mathrm{~Hz}, 1 \mathrm{H}), 7.15-7.36$ (m, $15 \mathrm{H}) ;{ }^{13} \mathrm{C}$ NMR $\left(\mathrm{CDCl}_{3}, 125 \mathrm{MHz}\right): \delta$ 56.0, 68.1, 82.6, 83.8, 109.2, 113.3, 114.1, 121.2, 126.3, 126.9, 127.3, 127.6, 128.0, 128.2, 128.3, 128.5, 140.0, 140.6, 142.1, 143.0, 152.6, 154.0, 199.7; HRMS $\left(\mathrm{M}^{+}\right)$found 458.1723, calcd 458.1729 for $\mathrm{C}_{28} \mathrm{H}_{26} \mathrm{O}_{6}$; HPLC (DAICEL 
Chiralpack AD, 10\% EtOH/hexane, flow rate $1.0 \mathrm{~mL} / \mathrm{min}): \mathrm{t}_{\mathrm{R}}=43.0$ (minor). $\mathrm{t}_{\mathrm{R}}=47.7$ (major).<smiles>COc1ccc(OC)c(C(=O)[C@H](O)[C@H](O)c2ccc3ccccc3c2)c1</smiles>

2-(Benzhydryloxy)-1-(2,5-dimethoxyphenyl)-3-hydroxy-3-(naphthalen-2-yl)propan1-one. 8 (R=2-naphthyl) Data are: ${ }^{1} \mathrm{H}$ NMR $\left(\mathrm{CDCl}_{3}, 500 \mathrm{MHz}\right): \delta 3.23(\mathrm{~d}, J=8.0 \mathrm{~Hz}, 1$ H), 3.57 (s, 3 H), 3.71 (s, 3 H), 5.18 (bs, 1 H), 5.39 (d, J = 2.5 Hz, 1 H), 5.48 (s, 1 H), 6.74-6.80 (m, $3 \mathrm{H}), 6.92-6.96$ (m, $3 \mathrm{H}), 7.09-7.35$ (m, $8 \mathrm{H})$, 7.45-7.48 (m, $2 \mathrm{H})$, 7.71-7.76 (m, $3 \mathrm{H}), 7.80-7.82$ (m, $1 \mathrm{H}) ;{ }^{13} \mathrm{C} \mathrm{NMR}\left(\mathrm{CDCl}_{3}, 125 \mathrm{MHz}\right): \delta$ 55.9, 56.0, 74.1, 82.7, 84.7, 113.3, 114.3, 120.9, 124.3, 125.3, 126.0, 126.2, 127.2, 127.3, 127.5, 127.75, 127.84, 127.9, 128.2, 128.3, 133.1, 133.3, 138.6, 140.4, 142.1, 152.5, 154.0, 200.2; HRMS $\left(\mathrm{FAB}^{+}\right.$) found 541.1998, calcd 541.1991 for $\mathrm{C}_{34} \mathrm{H}_{30} \mathrm{O}_{5} \mathrm{Na}$; HPLC (DAICEL Chiralpack $\mathrm{AD}, 10 \% \mathrm{EtOH} /$ hexane, flow rate $1.0 \mathrm{~mL} / \mathrm{min}$ ): $\mathrm{t}_{\mathrm{R}}=31.8$ (minor). $\mathrm{t}_{\mathrm{R}}=41.4$ (major).

\section{Product elaboration and absolute configuration determination}

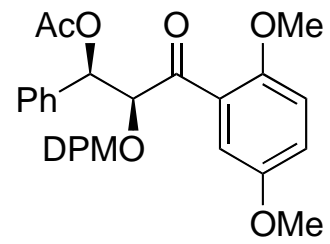

2-(Benzhydryloxy)-3-(2,5-dimethoxyphenyl)-3-oxo-1-phenylpropyl acetate. Ac-8 (R=Ph) To a solution of 2-(benzhydryloxy)-1-(2,5-dimethoxyphenyl)-3-hydroxy-3phenylpropan-1-one 8 (0.6 g, $1.28 \mathrm{mmol})$ in EtOAc $(3 \mathrm{~mL})$ were added pyridine $(0.31$ $\mathrm{mL}, 3.84 \mathrm{mmol})$, DMAP (20 mg), and acetyl chloride $(0.27 \mathrm{~mL}, 3.84 \mathrm{mmol})$ in an icewater bath under a nitrogen atmosphere. The mixture was stirred at $0{ }^{\circ} \mathrm{C}$ for $30 \mathrm{~min}$ and then stirred at ambient temperature for overnight. The reaction was quenched by $1 \mathrm{M} \mathrm{HCl}$. Then, it was poured into water and extracted with EtOAc. The organic phase was washed with water and brine, dried over $\mathrm{Na}_{2} \mathrm{SO}_{4}$, filtered, and evaporated in vacuo. The product (0.64 g, 97\%) was used for the next step without further purification, although it can be recrystallized with ethyl acetate and hexanes. Data are: ${ }^{1} \mathrm{H}$ NMR $\left(\mathrm{CDCl}_{3}, 500 \mathrm{MHz}\right): \delta$ 2.04 (s, 3 H), 3.59 (s, 3 H), 3.74 (s, 3 H), 5.26 (d, $J=3.5$ Hz, 1 H), 5.48 (s, 1 H), 6.14 (d, $J=3.5$ Hz, 1 H), 6.79-6.85 (m, 3 H), 6.96-6.99 (m, 1 H), 7.03 (d, $J=3.0$ Hz, 1 H), 7.10$7.32(\mathrm{~m}, 13 \mathrm{H}) ;{ }^{13} \mathrm{C} \mathrm{NMR}\left(\mathrm{CDCl}_{3}, 75 \mathrm{MHz}\right): \delta 21.2,56.0,56.1,75.5,83.1,83.5,113.4$, $114.5,120.6,127.1,127.2,127.4,127.8,128.0,128.2,128.36,128.38,128.5,137.6$, 141.1, 142.6, 152.4, 154.1, 170.0, 198.9; HRMS (FAB $\left.{ }^{+}\right)$found 533.1953, calcd 533.1940 for $\mathrm{C}_{32} \mathrm{H}_{30} \mathrm{O}_{6} \mathrm{Na}$. 
<smiles>COc1ccc(OC)c(C(=O)C(O)C(OC(C)=O)c2ccccc2)c1</smiles>

3-(2,5-Dimethoxyphenyl)-2-hydroxy-3-oxo-1-phenylpropyl acetate (10). A solution of 2-(benzhydryloxy)-3-(2,5-dimethoxyphenyl)-3-oxo-1-phenylpropyl acetate (0.51 g, 1 mmole) in $25 \mathrm{~mL} \mathrm{CH}_{2} \mathrm{Cl}_{2}$ was cooled to $-78^{\circ} \mathrm{C}$ and $\mathrm{TiCl}_{4}\left(1.1 \mathrm{~mL}, 1.0 \mathrm{M} \mathrm{CH}_{2} \mathrm{Cl}_{2}\right)$ was added drop-wisely. The resulting dark red solution was stirred at $-78^{\circ} \mathrm{C}$ for 2 hours. The reaction was then quenched by saturated aqueous $\mathrm{NaHCO}_{3}$ solution. The aqueous layer was extracted with ether. The combined organic layers were washed with water and brine, then dried over $\mathrm{MgSO}_{4}$, filtered and concentrated in vacuo. Radial chromatography afforded the desired compound (0.27 g, 77\%). Data are: ${ }^{1} \mathrm{H} \mathrm{NMR}\left(\mathrm{CDCl}_{3}, 500 \mathrm{MHz}\right): \delta$ 1.99 (s, 3 H), 3.81 (s, 3 H), 3.95 (s, 3 H), 3.97 (d, $J=7.0$ Hz, 1 H), 5.44 (dd, J = 1.5, 7.0 $\mathrm{Hz}, 1 \mathrm{H}), 6.13$ (d, $J=1.5 \mathrm{~Hz}, 1 \mathrm{H}$ ), 6.98 (d, $J=9.0 \mathrm{~Hz}, 1 \mathrm{H}$ ), 7.13 (dd, $J=3.0,9.0 \mathrm{~Hz}, 1$ $\mathrm{H})$, 7.27-7.44 (m, $6 \mathrm{H}) ;{ }^{13} \mathrm{C} \mathrm{NMR}\left(\mathrm{CDCl}_{3}, 125 \mathrm{MHz}\right): \delta 20.9$, 56.1, 56.3, 75.0, 79.3, 113.5, 114.8, 122.3, 127.0, 128.3, 128.6, 137.9, 153.6, 154.1, 169.8, 199.2; HRMS $\left(\mathrm{FAB}^{+}\right)$found 367.1156, calcd 367.1158 for $\mathrm{C}_{19} \mathrm{H}_{20} \mathrm{O}_{6} \mathrm{Na}$.<smiles>COc1ccc(OC)c(OC(=O)[C@H](O)[C@H](OC(C)=O)c2ccccc2)c1</smiles>

2,5-Dimethoxyphenyl 3-acetoxy-2-hydroxy-3-phenylpropanoate (11). To a flame dried round bottom flask was added activated 4 angstrom molecular sieves (1.0 g), $\mathrm{K}_{2} \mathrm{CO}_{3}(0.2142$ g, $1.55 \mathrm{mmol}$ ), trans-N,N-bis(p-toluenesulfonyl)-1,2-cyclohexanediamine ( $0.3275 \mathrm{~g}, 0.775 \mathrm{mmol})$ and $10 \mathrm{~mL}$ of $\mathrm{CH}_{2} \mathrm{Cl}_{2}$. The mixture was cooled to $0^{\circ} \mathrm{C}$ and $\mathrm{SnCl}_{4}$ (0.78 mL, $1 \mathrm{M}$ in $\mathrm{CH}_{2} \mathrm{Cl}_{2}$ ) was added followed by bis(trimethylsilyl) peroxide (0.33 mL, $1.55 \mathrm{mmol})$. The mixture was stirred at $0^{\circ} \mathrm{C}$ for 15 minutes, then 3-(2,5-dimethoxyphenyl) -2-hydroxy-3-oxo-1-phenylpropyl acetate 10 (0.2669 g, $0.775 \mathrm{mmol}$ ) was added as a $\mathrm{CH}_{2} \mathrm{Cl}_{2}$ solution $(7.5 \mathrm{~mL})$. The reaction stirred at $0^{\circ} \mathrm{C}$ for $50 \mathrm{~min}$, at which time the reaction was quenched by the addition of a saturated aqueous $\mathrm{NaHCO}_{3}$ followed by a saturated aqueous $\mathrm{Na}_{2} \mathrm{~S}_{2} \mathrm{O}_{3}$ solution. The mixture was stirred at ambient temperature for 30 minutes then diluted with EtOAc and the layers separated. The aqueous phase was extracted with EtOAc and the combined organic layers were washed with $\mathrm{H}_{2} \mathrm{O}$ and brine. The mixture was then filtered, concentrated and purified via radial chromatography to provide the desired product (0.22 g, 79\%). Data are: ${ }^{1} \mathrm{H}$ NMR $\left(\mathrm{CDCl}_{3}, 500 \mathrm{MHz}\right): \delta 2.15$ (s, $3 \mathrm{H}$ ), 3.05 (d, $J=8.5 \mathrm{~Hz}, 1 \mathrm{H}$ ), 3.747 (s, $3 \mathrm{H}$ ), 3.752 (s, $3 \mathrm{H}$ ), 4.70 (dd, $J=3.0,8.5 \mathrm{~Hz}$, 1H), 6.30 (d, J= 3.0 Hz, 1H), 6.59 (d, J=3.0 Hz, 1H), 6.76 (dd, J= 3.0, 9.5 Hz, 1H), 6.90 (d, $J=8.5 \mathrm{~Hz}, 1 \mathrm{H}), 7.32-7.48$ (m, $5 \mathrm{H}) ;{ }^{13} \mathrm{C} \mathrm{NMR}\left(\mathrm{CDCl}_{3}, 125 \mathrm{MHz}\right): \delta 21.1,56.0,56.6$, 73.9, 75.7, 109.3, 112.3, 113.6, 127.2, 128.7, 128.8, 136.6, 139.5, 145.3, 153.9, 169.8, 170.0; HRMS $\left(\mathrm{M}^{+}\right)$found 360.1223, calcd 360.1209 for $\mathrm{C}_{19} \mathrm{H}_{20} \mathrm{O}_{7}$. 
<smiles>COC(=O)[C@H](O)[C@H](O)c1ccccc1</smiles>

Methyl 2,3-dihydroxy-3-phenylpropanoate (12). To a flame dried round bottom flask containing 2,5-dimethoxyphenyl 3-acetoxy-2-hydroxy-3-phenylpropanoate 11 (0.1 g, $0.28 \mathrm{mmol}$ ) was added $2.8 \mathrm{~mL}$ of $\mathrm{MeOH}$ and $2.8 \mathrm{~mL}$ of THF. The solution was cooled to $0^{\circ} \mathrm{C}$ and then a freshly prepared $\mathrm{NaOMe} / \mathrm{MeOH}$ solution $(5.8 \mathrm{~mL}, 0.1 \mathrm{M}$ ) was added. The solution stirred at $0^{\circ} \mathrm{C}$ for $1 \mathrm{~h}$ then at ambient temperature for $17 \mathrm{~h}$ at which time the reaction was quenched by the addition of a saturated aqueous $\mathrm{NH}_{4} \mathrm{Cl}$ solution. The resulting solution was extracted with $\mathrm{Et}_{2} \mathrm{O}$ and the combined organic layers were dried over $\mathrm{MgSO}_{4}$, filtered and concentrated. The crude residue was purified via flash column chromatography to provide the title compound $(0.0182 \mathrm{~g}, 85 \%)$. Data are: $[\alpha]_{\mathrm{D}}^{23}-7.6$ (c 0.3, $\mathrm{CHCl}_{3}$ ); [Lit. -10.7 (c 1.0, $\left.\mathrm{CHCl}_{3}\right)$ ]; ${ }^{1} \mathrm{H} \mathrm{NMR}\left(\mathrm{CDCl}_{3}, 500 \mathrm{MHz}\right): \delta 2.83$ (d, $J=6.5$ Hz, 1 H), 3.17 (d, $J=5.5$ Hz, 1 H), 3.80 (s, 3 H), 4.37 (dd, $J=3.0,6.5$ Hz, 1 H), 5.01 (dd, $J=3.0,6.5 \mathrm{~Hz}, 1 \mathrm{H}), 7.30-7.41$ (m, $5 \mathrm{H}) ;{ }^{13} \mathrm{C} \mathrm{NMR}\left(\mathrm{CDCl}_{3}, 125 \mathrm{MHz}\right): \delta$ 53.1, 74.6, 74.9, 126.4, 128.3, 128.7, 140.1, 173.4; HRMS (FAB ${ }^{+}$) found 219.0635, calcd 219.0633 for $\mathrm{C}_{10} \mathrm{H}_{12} \mathrm{O}_{4} \mathrm{Na}$.

\section{NMR Spectra}


S-11
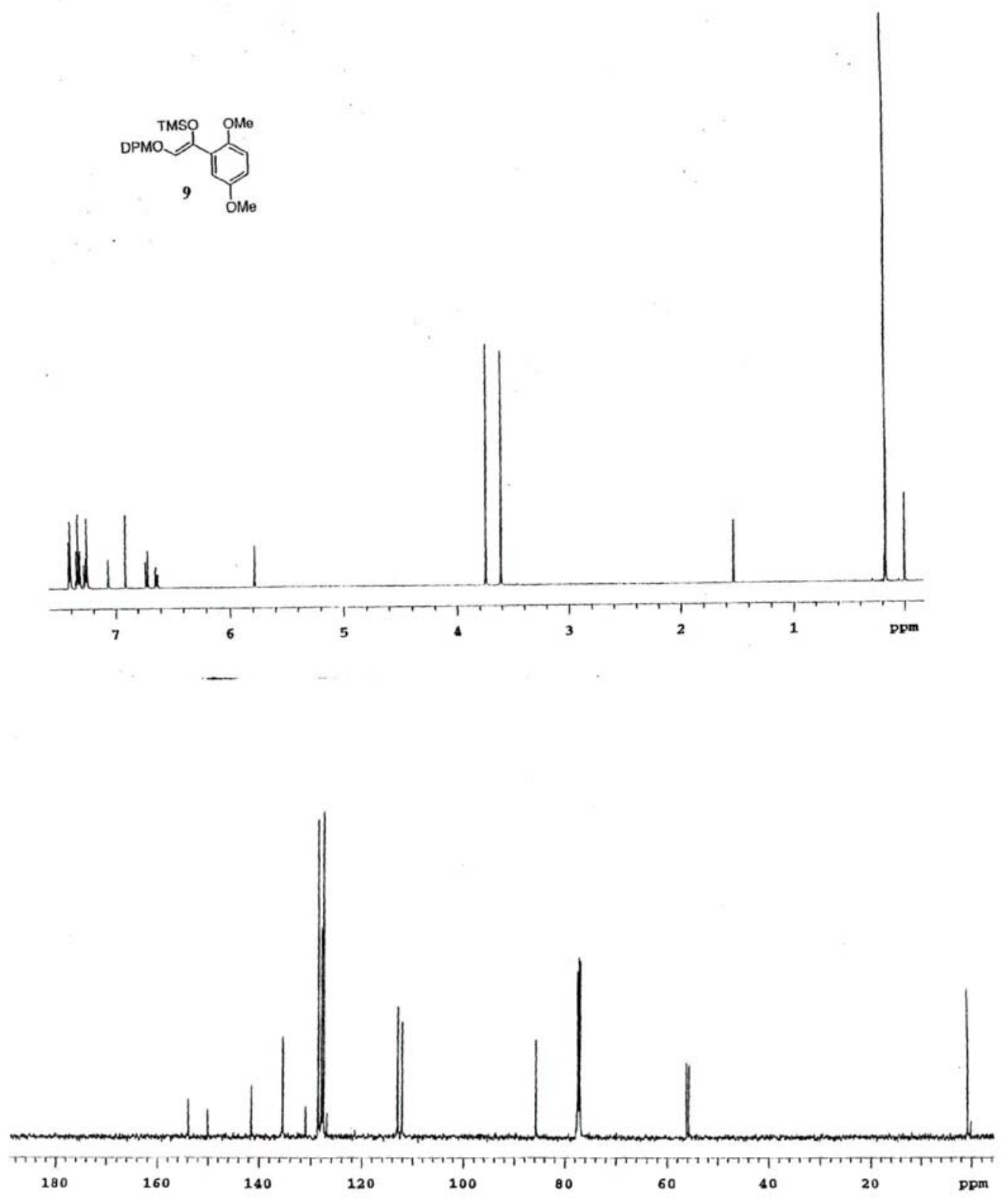
S-12
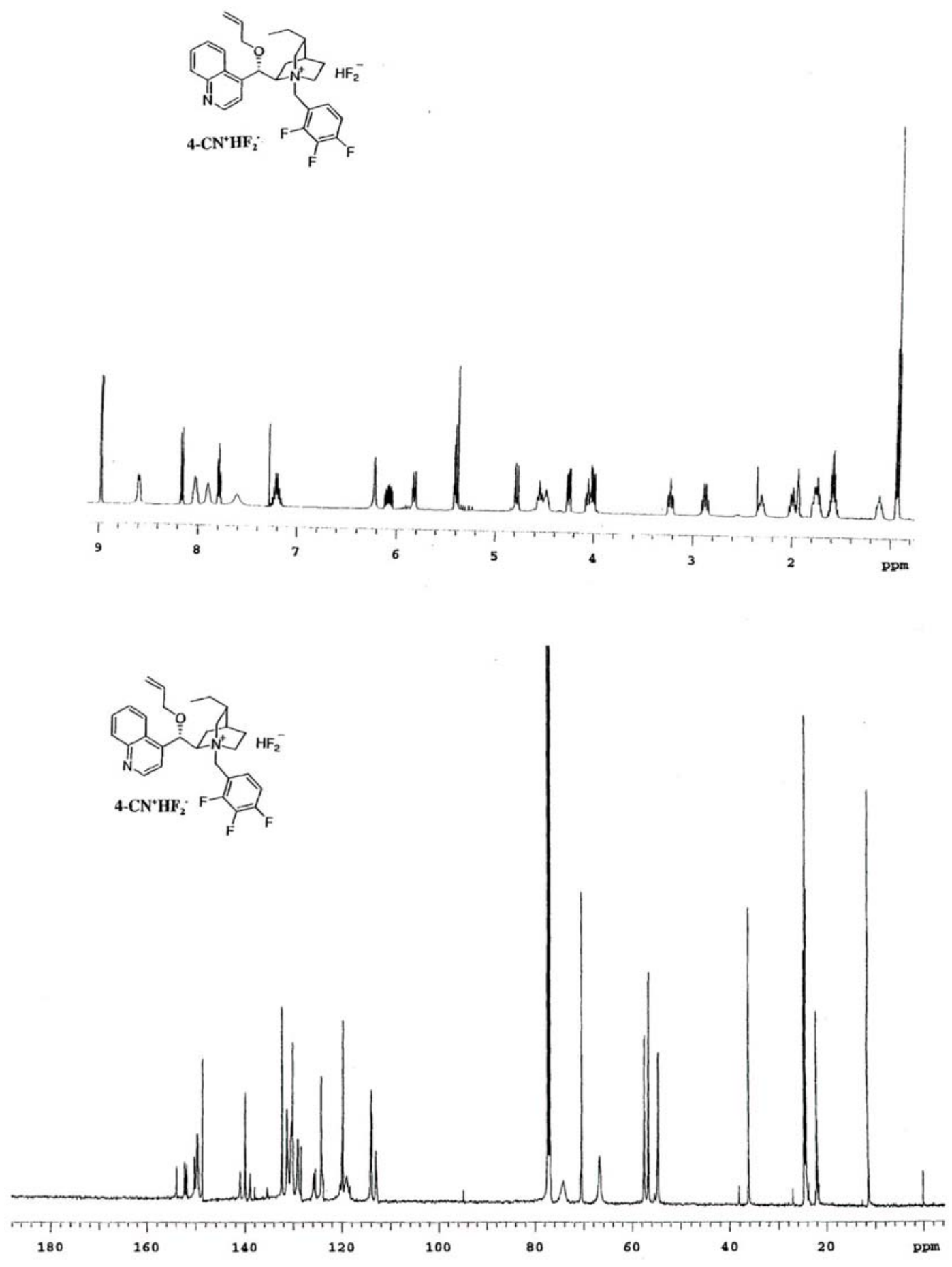
S-13
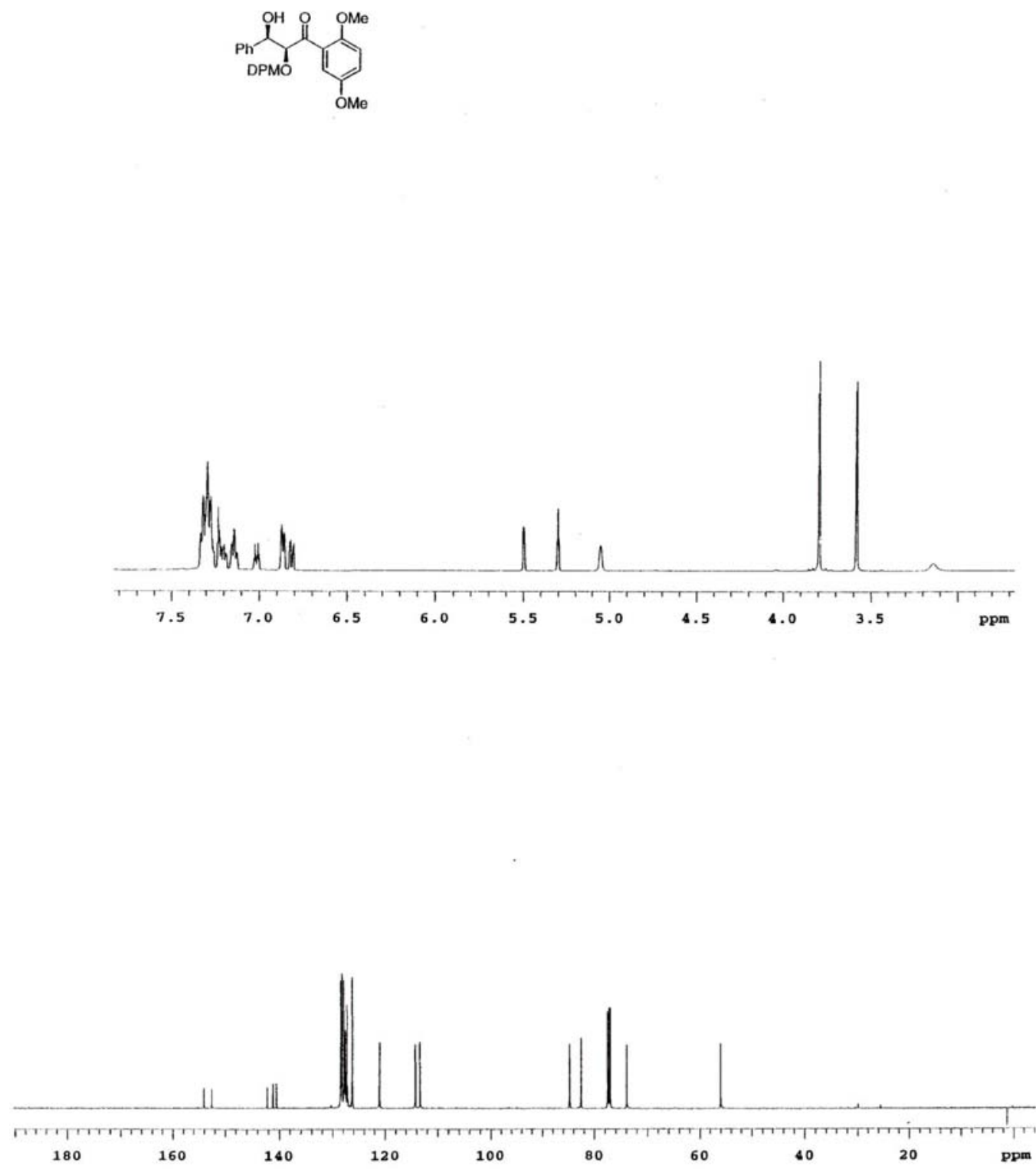

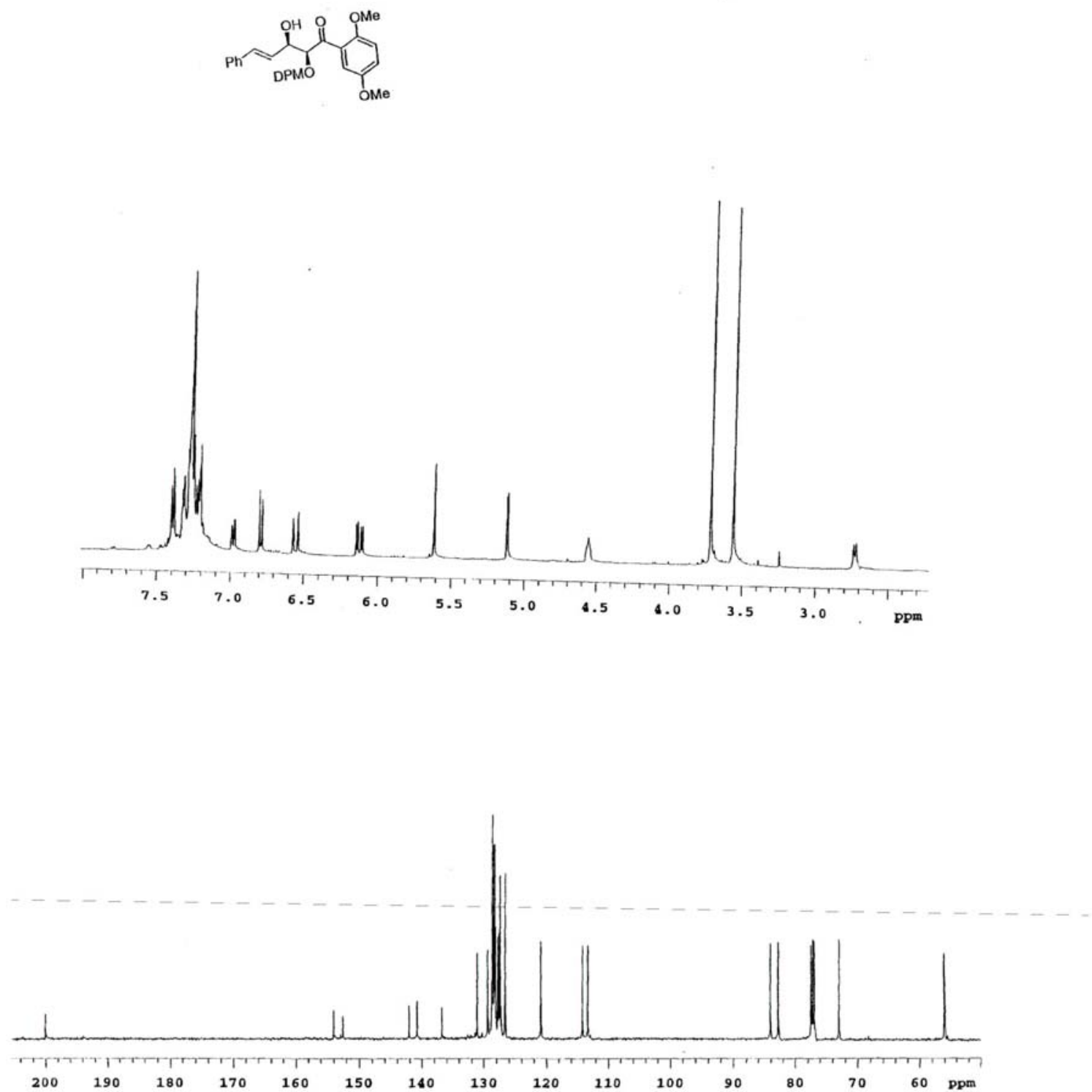

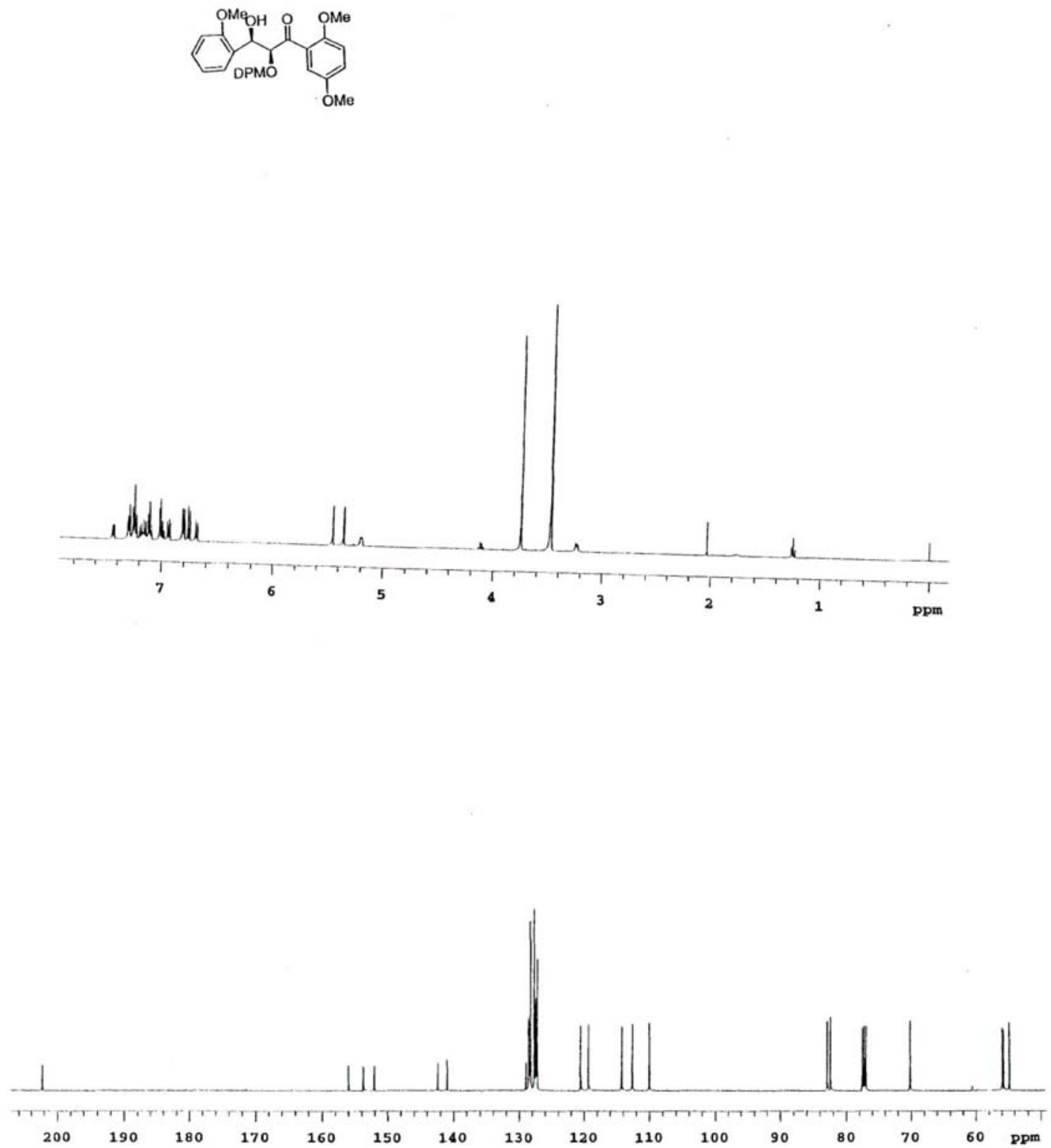
S-16
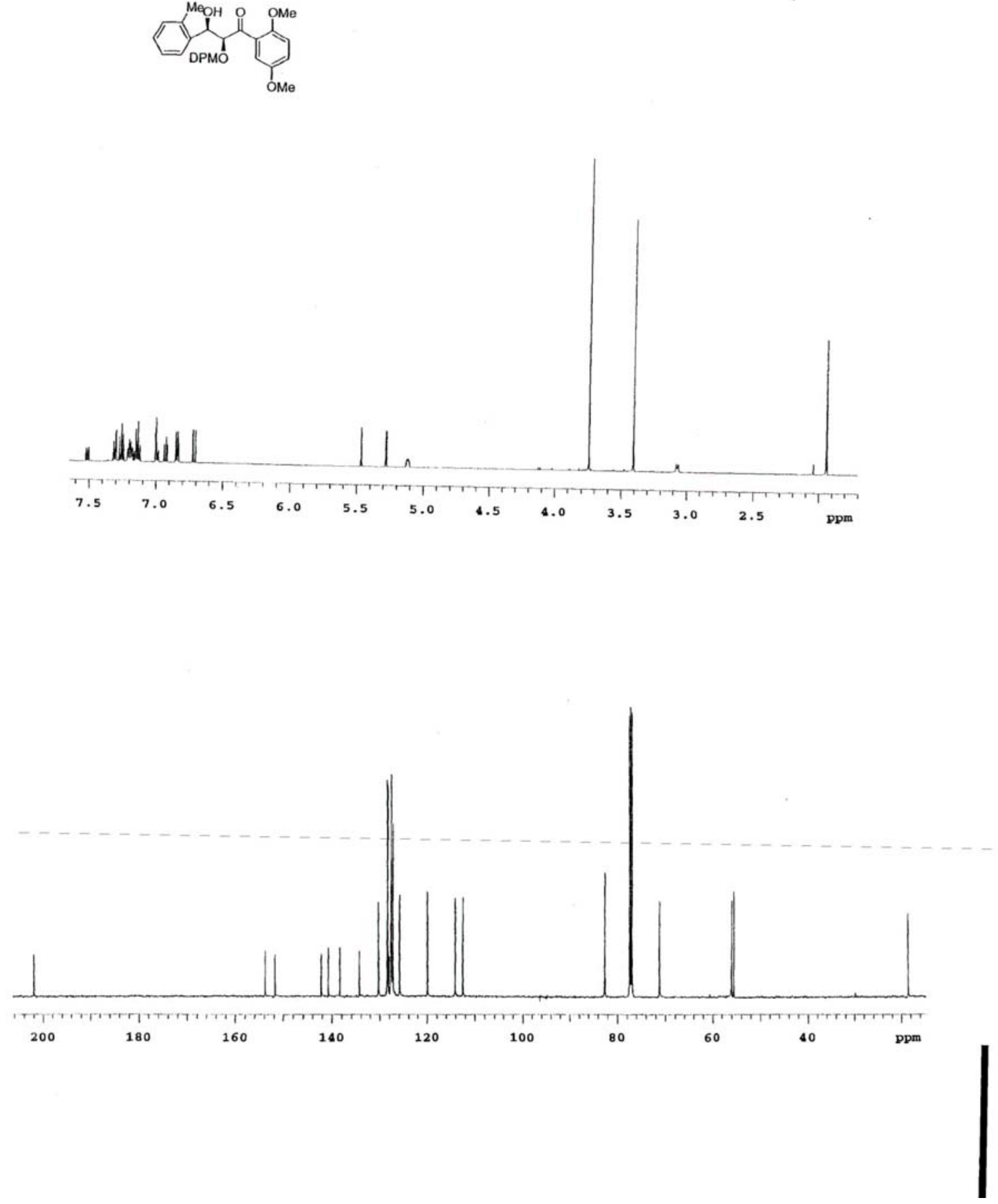

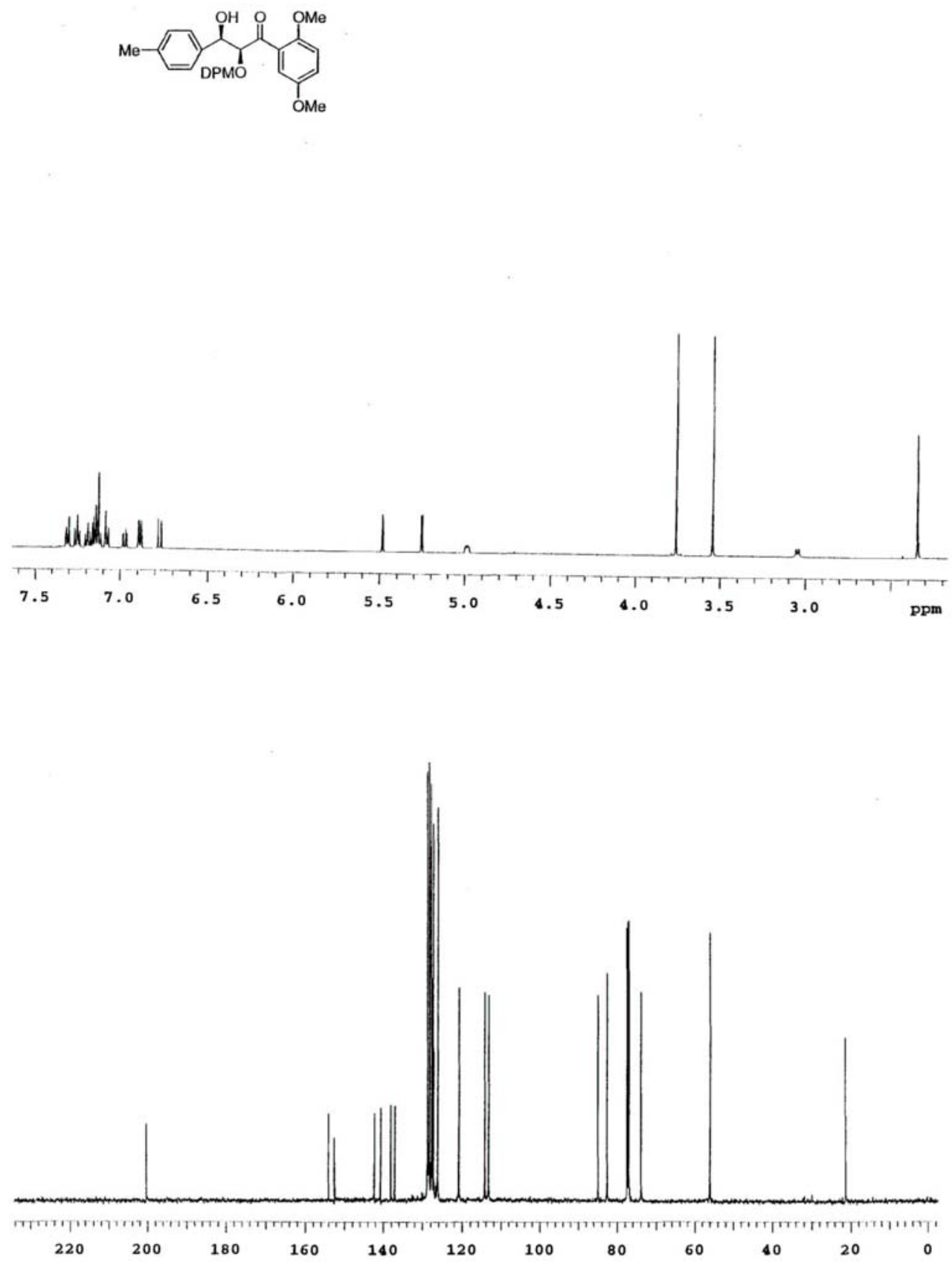

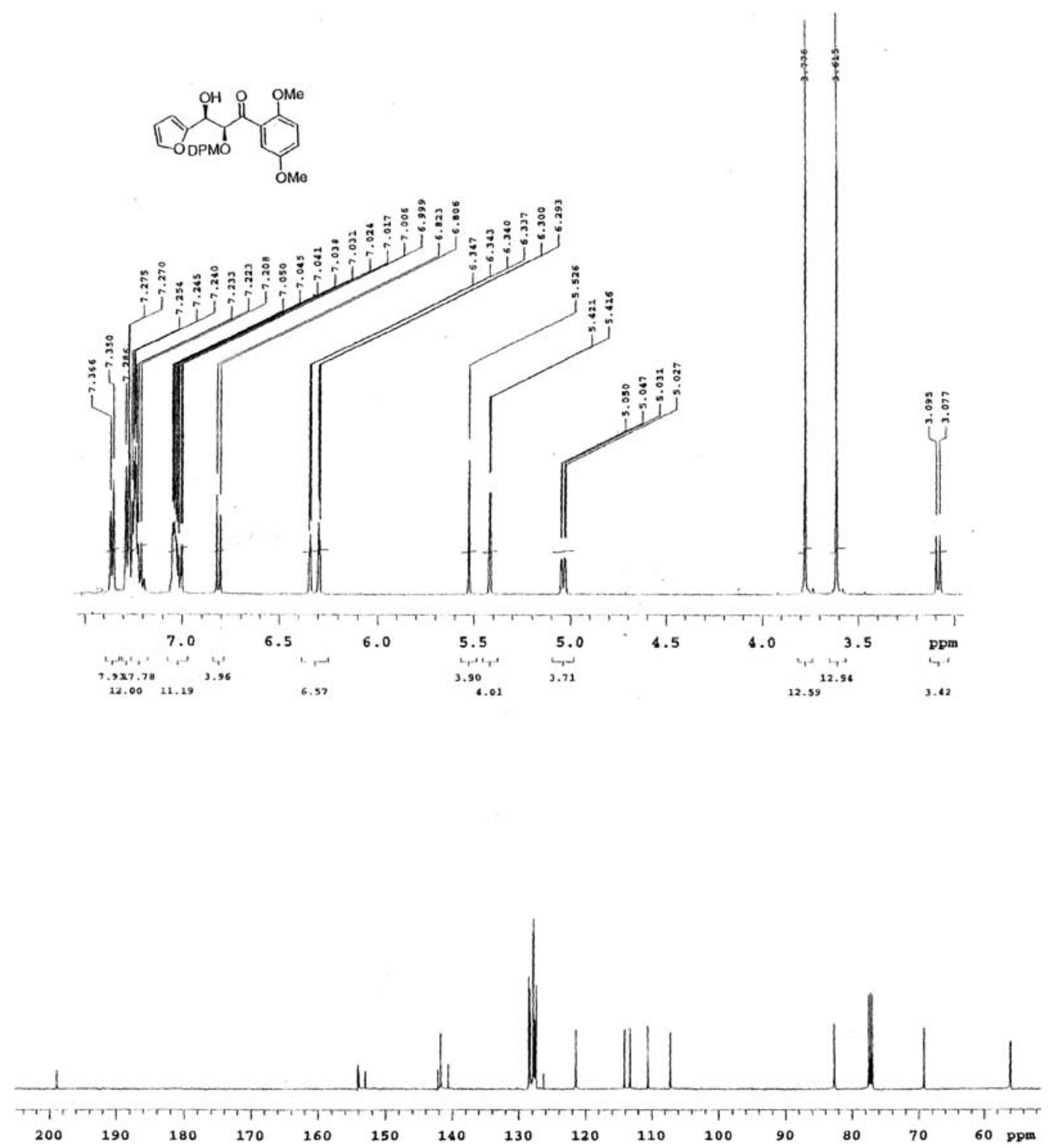

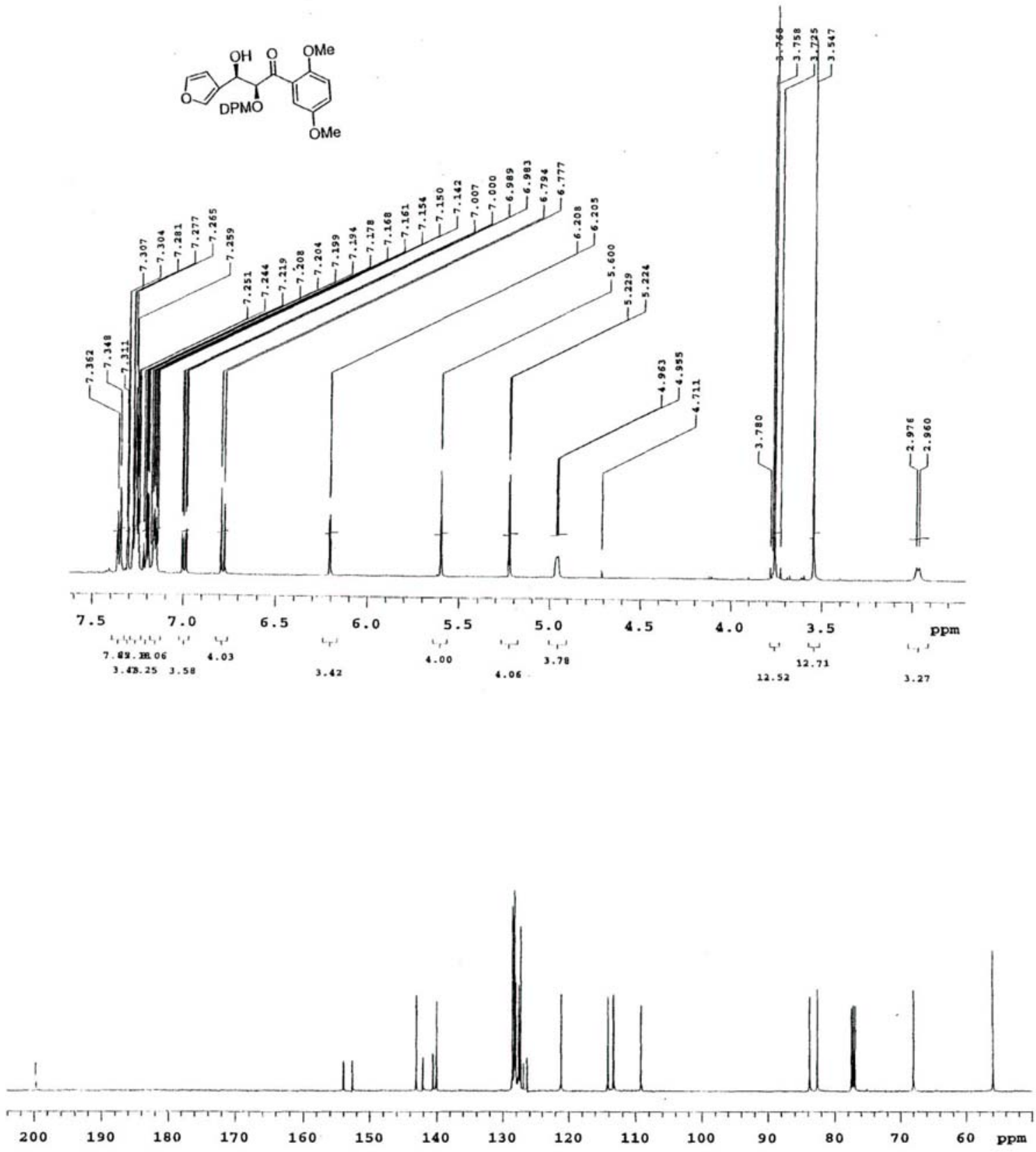

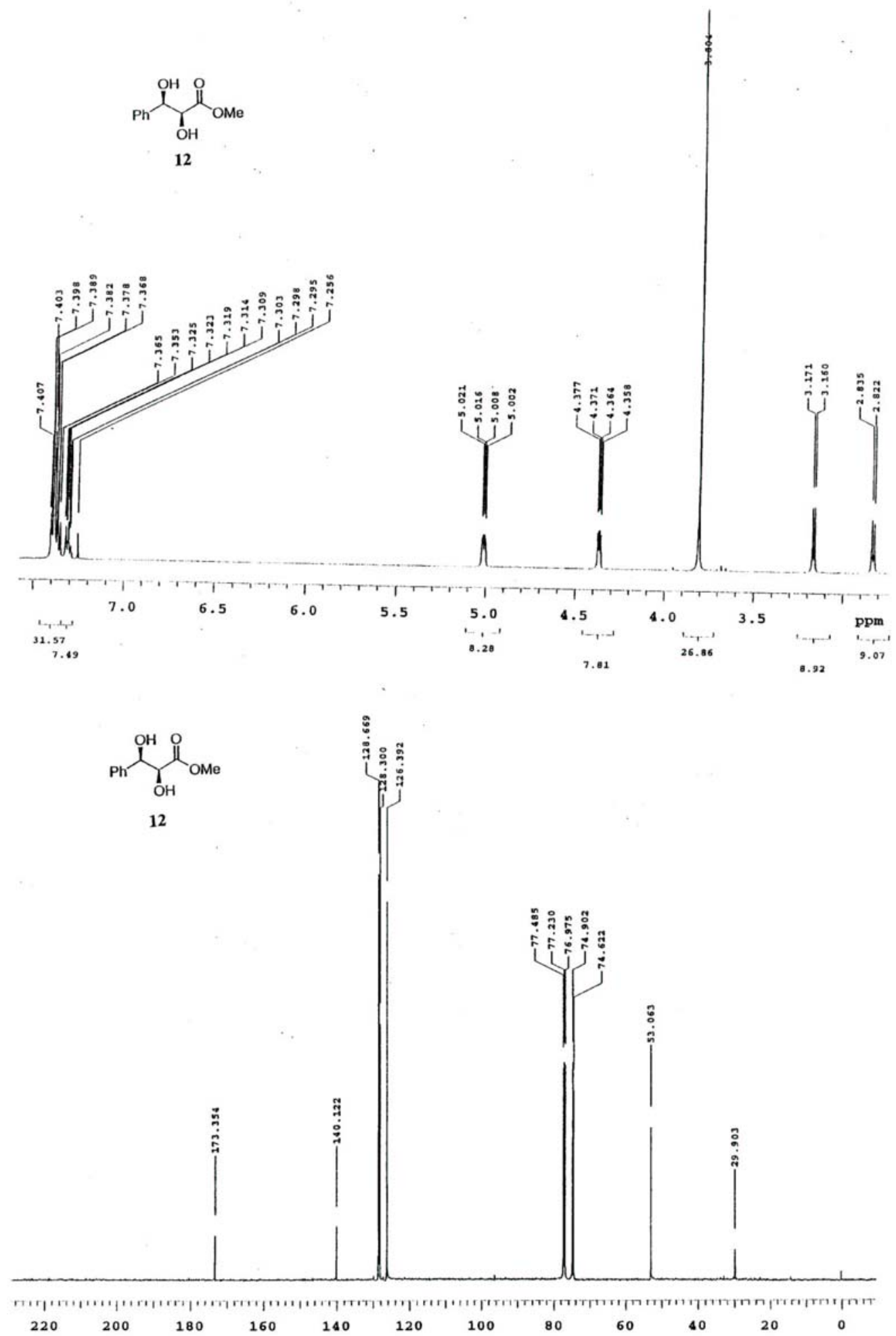

HPLC Data: Cmpd 8 (R=Ph) Resolved (Asymmetric PTC aldol product) 
Date: Mon, Mar 29, 2004 14:41

Data: Untitled-1 8FEB 1 04-001

Sample: jl\#256

$\mathrm{ADD}$ Column; 10\% EtOH/Hexane

$1.0 \mathrm{ml} / \mathrm{min}, 320 \mathrm{psi}$

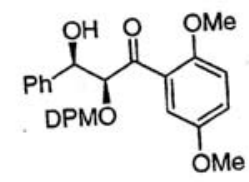

Method: ptcy

Sampling Int: 0.1 Seconds

Data:

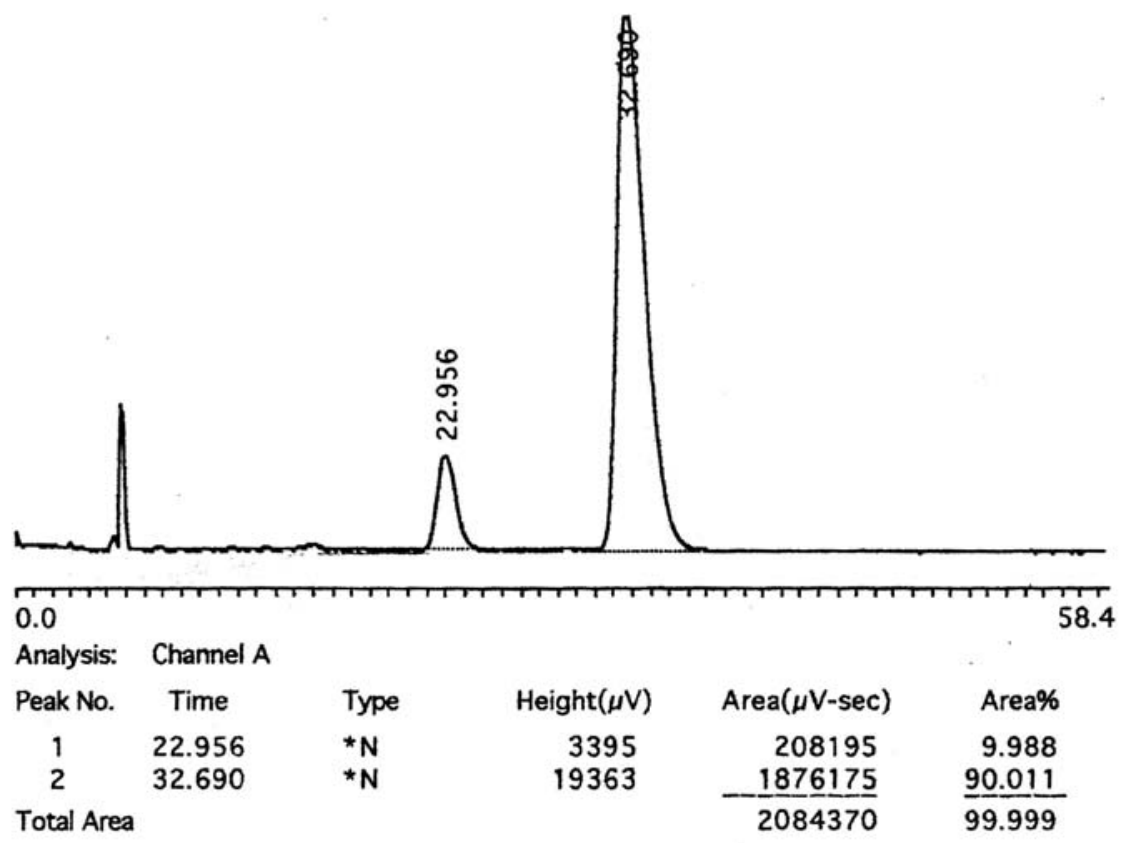

Cmpd 8 (R=Ph) Racemic 
Date: Fri, Feb 6, 2004 0:17

Data: ptcy-05FEB 104-001
Sample: jl\#237 (BA racemic repeat) D ADO Column; 10\% EtOH/Hexane
$1 \mathrm{ml} / \mathrm{min}, 320$ psi

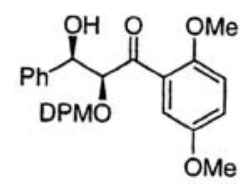

Method: ptcy

Sampling Int: 0.1 Seconds

Data:

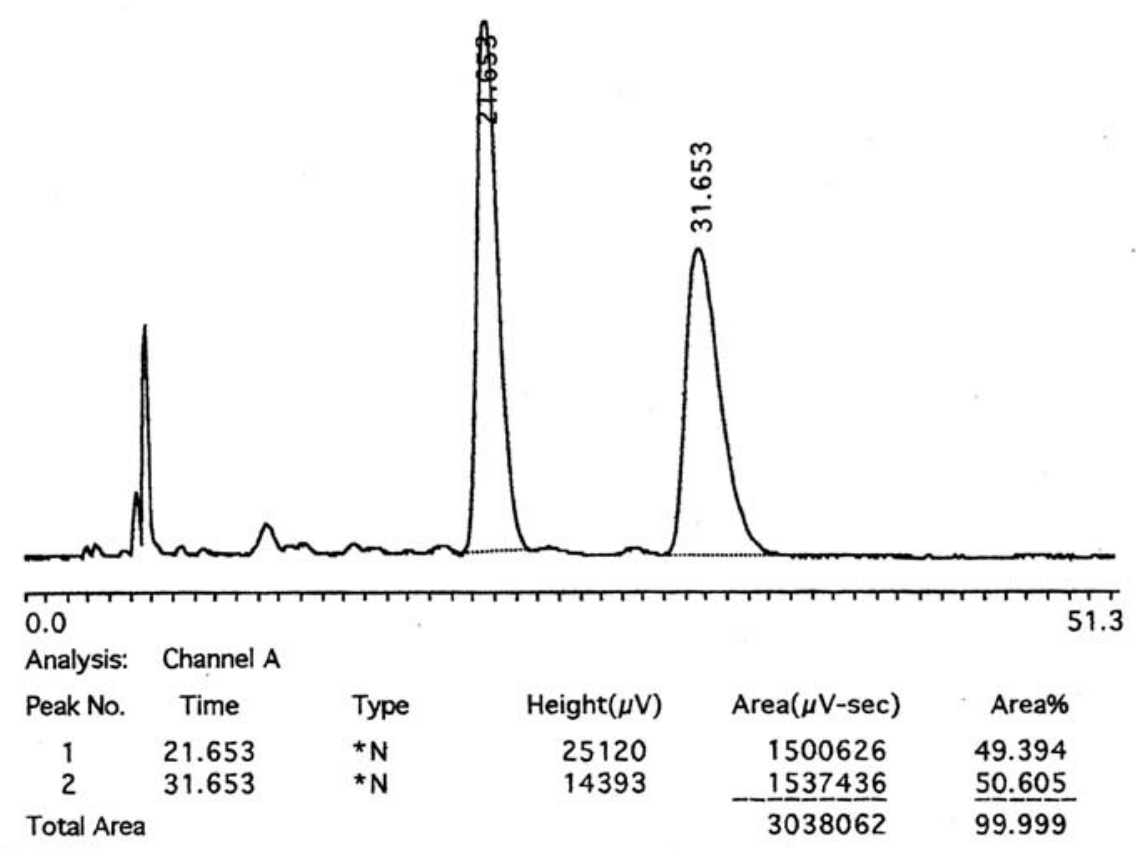

Cmpd 8 (R=2-biphenyl) Resolved 
Date: Thu, Sep 16, 2004 13:47

Data: jIII-24-\#2

Sample: jIII-24-\#2 $\mathrm{ADO}$ Column; $10 \% \mathrm{EtOH} /$ Hexane $1.0 \mathrm{ml} / \mathrm{min}, 330000 \mathrm{psi}$

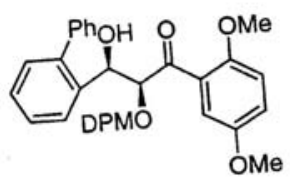

Processing File: ZFY-II-86

Method: ptcy

Sampling Int: 0.1 Seconds

Data:

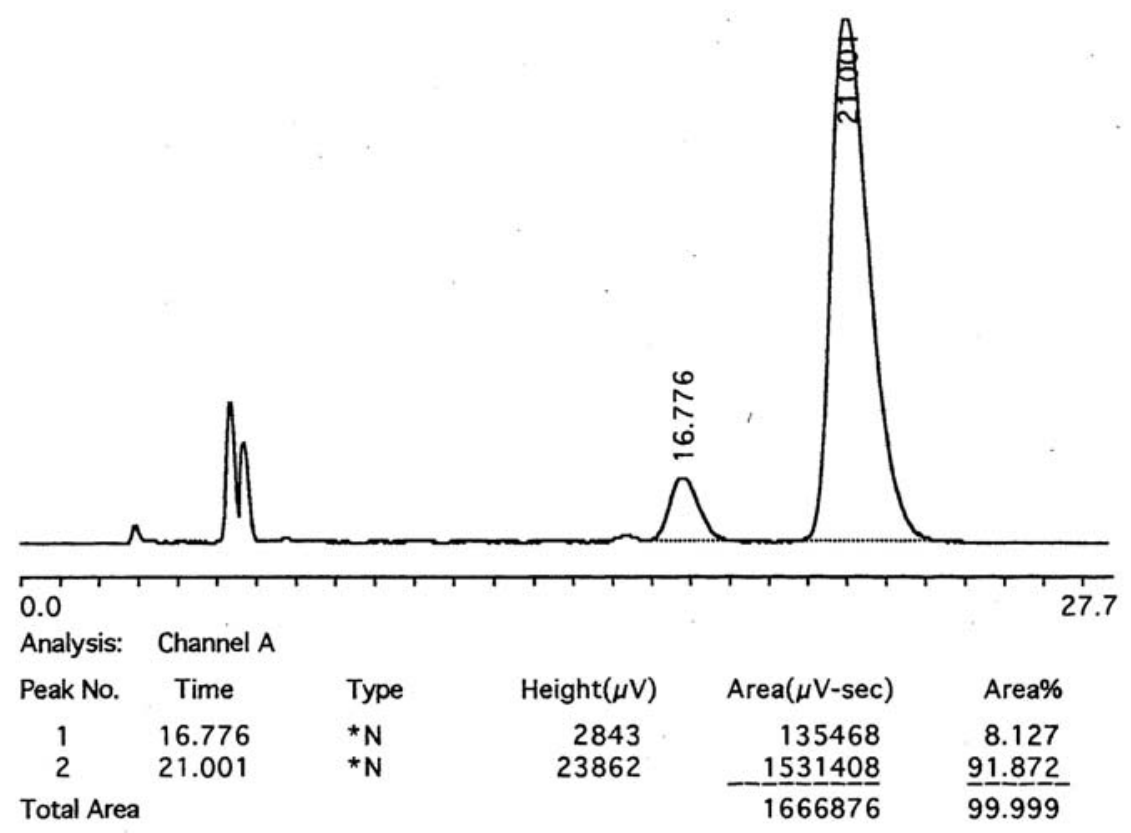


Cmpd 8 (R=2-biphenyl) Racemic

Date: Thu, Sep 16, 2004 12:47

Data: jIII-25-\#2

Sample: jill-25-\#2 $\mathrm{ADD}$ Column; $10 \% \mathrm{EtOH} /$ Hexane $1.0 \mathrm{ml} / \mathrm{min}, 3300000 \mathrm{psi}$

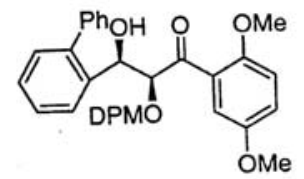

Processing File: ZFY-II-86

Method: ptcy

Sampling Int: 0.1 Seconds

Data:

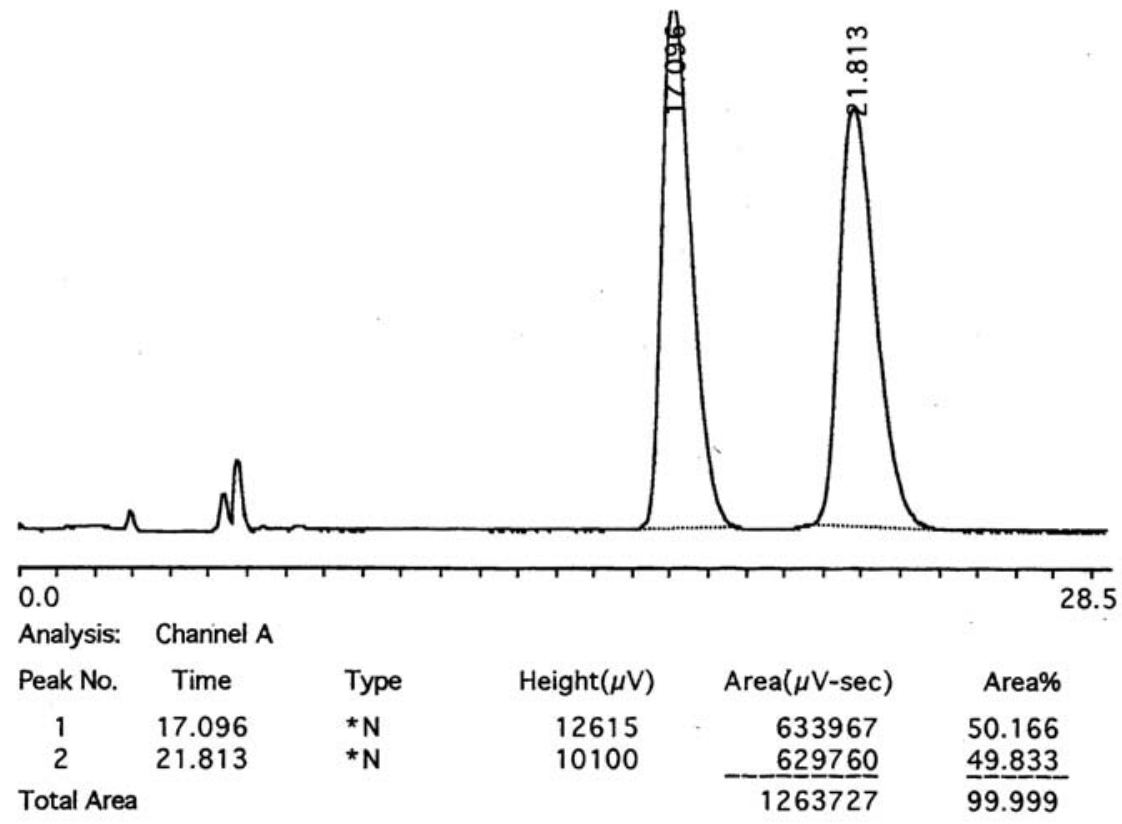


Cmpd 8 (R=2-naphthyl) Resolved

Date: Wed, May 12, 2004 17:22

Data: ptcy-12MAY104-001

Sample: ji\#279-1

ADD Column; $10 \%$ EtOH/Hexane

$1 \mathrm{ml} / \mathrm{min}, 340 \mathrm{psi}$

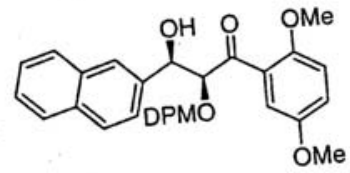

Method: ptcy

Sampling Int: 0.1 Seconds

Data:

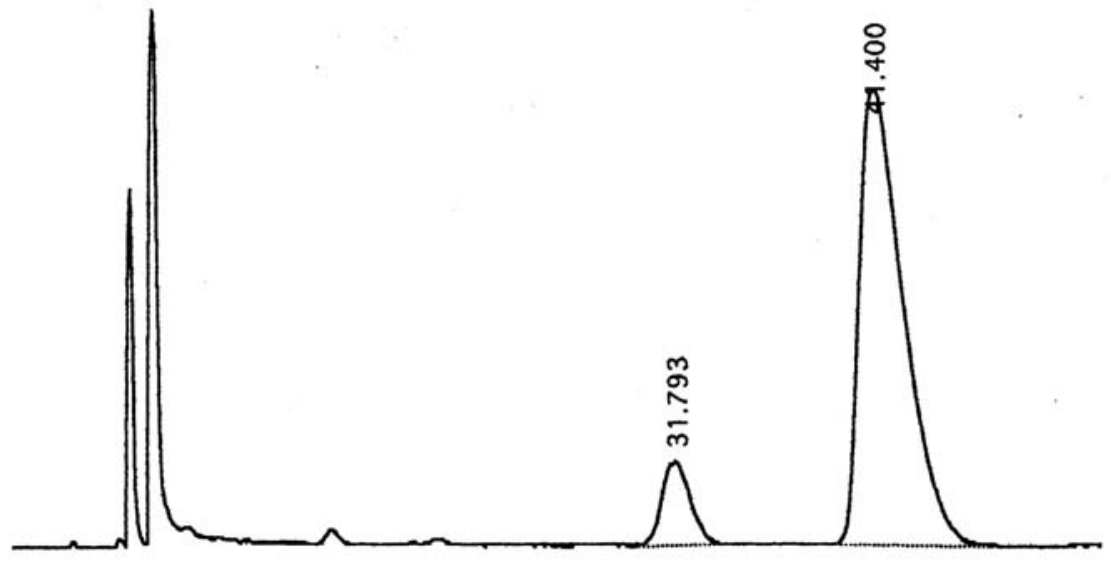

\begin{tabular}{lclccc} 
& & & & \\
Analysis: & Channel A & & & & \\
Peak No. & Time & Type & Height $(\mu \mathrm{V})$ & Area $(\mu \mathrm{V}$-sec $)$ & Area\% \\
1 & 31.793 & ${ }^{*} \mathrm{~N}$ & 9438 & 840532 & 10.461 \\
2 & 41.400 & ${ }^{N}$ & 51629 & -7194235 & 89.538 \\
\hline
\end{tabular}

Cmpd 8 (R=2-naphthyl) Racemic 
Date: Thu, May 6, 2004 18:58

Data: ptcy-06MAY104-001

Sample: jl\#273

ADD Column; 10\% EtOH/Hexane

$1.0 \mathrm{ml} / \mathrm{min}, 330 \mathrm{psi}$

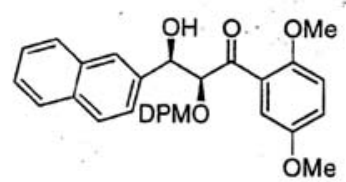

Method: ptcy

Sampling Int: 0.1 Seconds

Data:

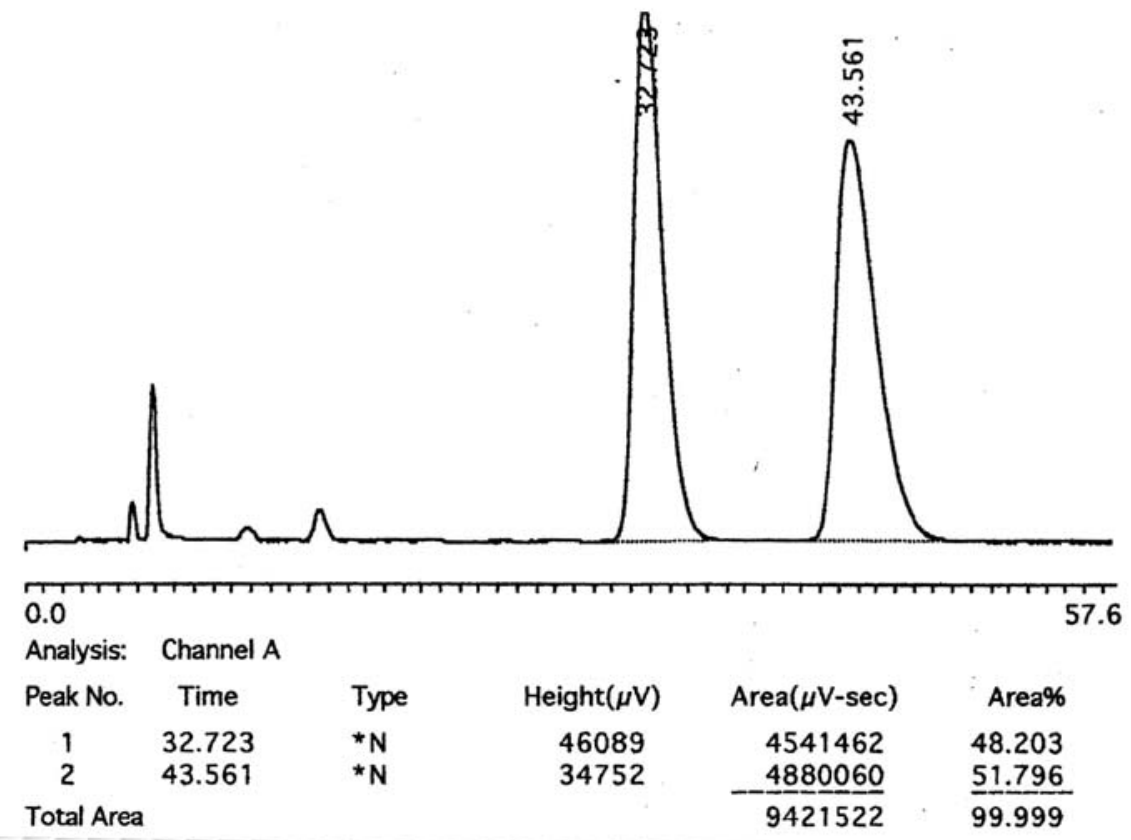


Cmpd 8 (R=2-styryl) Resolved

Date: Wed, May 12, 2004 21:00

Data: ptcy-12MAY104-002

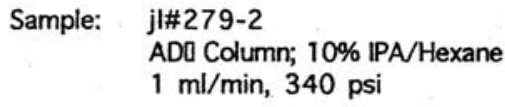

Method: ptcy

Sampling Int: 0.1 Seconds

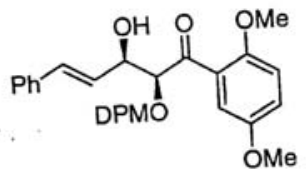

Data:

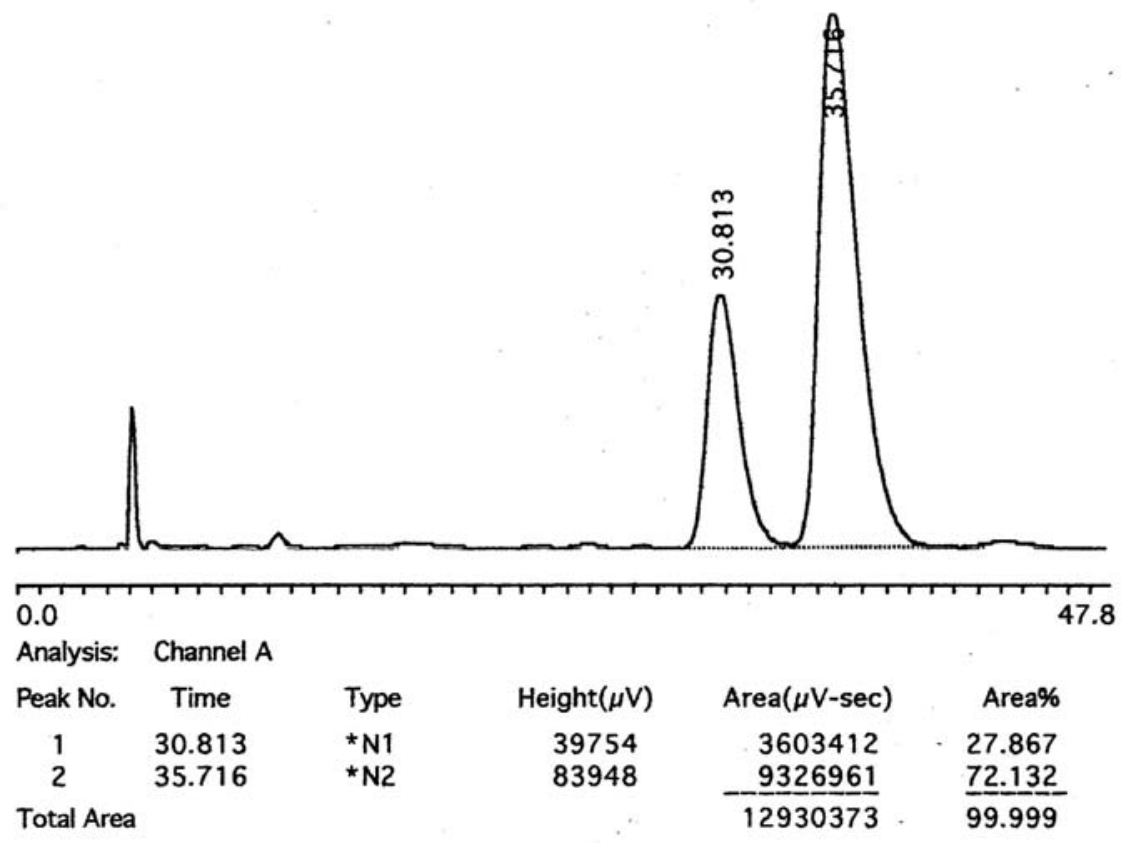


Cmpd 8 (R=2-styryl) Racemic

Date: Wed, May 12, 2004 20:11

Data: ptcy-12MAY104-002

Sample: jl\#275

ADD Column; 10\% IPA/Hexane

$1 \mathrm{ml} / \mathrm{min}, 340 \mathrm{psi}$

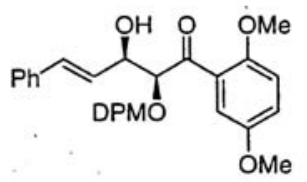

Method: ptcy

Sampling Int: 0.1 Seconds

Data:

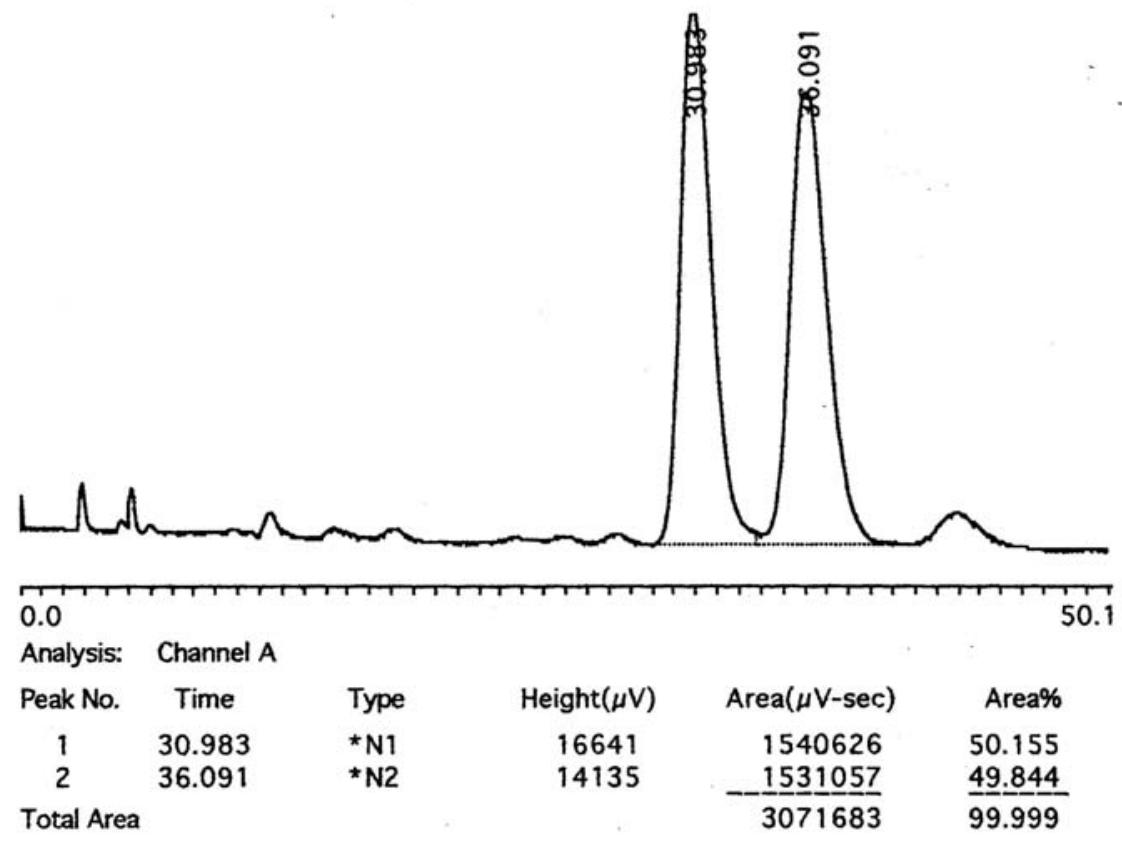




\section{X-ray Data}

Compound $8(\mathrm{R}=\mathrm{Ph})$. Deposited in Cambridge Crystallographic Data Centre (CCDC\#271201)

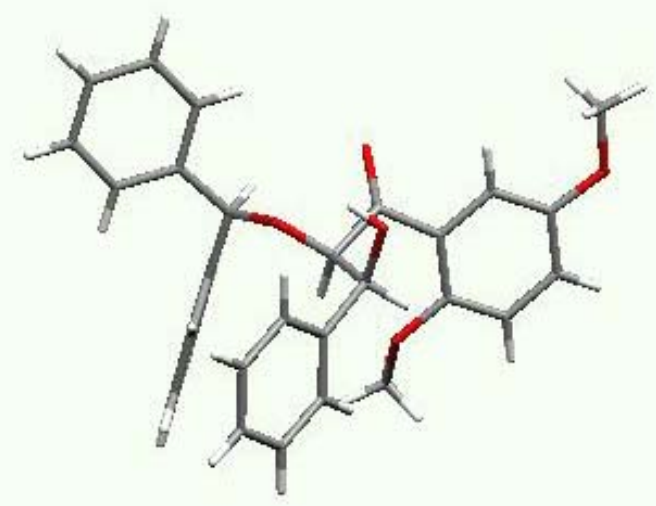

Table 1-S. Crystal data and structure refinement for cmpd 8 (R=Ph).

Identification code

Empirical formula

Formula weight

Temperature

Wavelength

Crystal system

Space group

Unit cell dimensions

Volume

Z

Density (calculated)

Absorption coefficient

$\mathrm{F}(000)$

Crystal size

Theta range for data collection

Index ranges jl0-cmpd $8(\mathrm{R}=\mathrm{Ph})$

C30 H28 O5

468.52

296(2) K

$0.71073 \AA$

Monoclinic

$\mathrm{P} 2(1) / \mathrm{C}$

$\mathrm{a}=12.8162(14) \AA \quad \alpha=90.00(10)^{\circ}$.

$\mathrm{b}=12.8977(11) \AA \quad \beta=109.913(7)^{\circ}$

$\mathrm{c}=16.3560(15) \AA \quad \gamma=90.00^{\circ}$

2542.0(4) $\AA^{3}$

4

$1.224 \mathrm{Mg} / \mathrm{m}^{3}$

$0.083 \mathrm{mu}$

992

$0.56 \times 0.53 \times 0.16 \mathrm{~mm}^{3}$

0.998 to $25.00^{\circ}$.

$-1<=\mathrm{h}<=15,-1<=\mathrm{k}<=15,-19<=\mathrm{l}<=18$ 
Reflections collected

Independent reflections

Completeness to theta $=25.00^{\circ}$

Absorption correction

Max. and min. transmission

Refinement method

Data / restraints / parameters

Goodness-of-fit on $\mathrm{F}^{2}$

Final R indices [I $>2$ sigma(I)]

$\mathrm{R}$ indices (all data)

Largest diff. peak and hole
4464

$2772[\mathrm{R}$ (int) $=2$

$99.8 \%$

none

0.5511 and 0.4815

Full-matrix least-squares on $\mathrm{F}^{2}$

4464 / 0 / 318

0.994

$\mathrm{R} 1=0.0511, \mathrm{wR} 2=0.1101$

$\mathrm{R} 1=0.0960, \mathrm{wR} 2=0.1293$

0.186 and -0.163 e. $\AA^{-3}$

Compound Acetyl-8 ( $\mathrm{R}=\mathrm{Ph})$. Deposited in Cambridge Crystallographic Data Centre (CCDC\#268187)

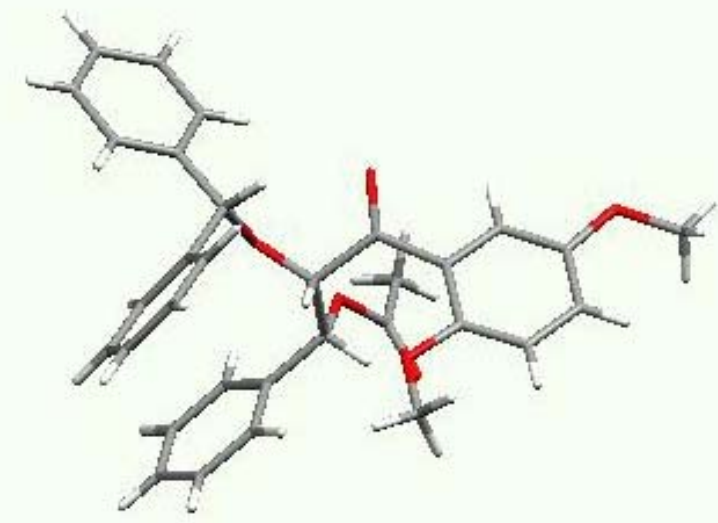

Table 2-S. Crystal data and structure refinement for Acetyl-8 (R=Ph).

Identification code

Empirical formula

Formula weight

Temperature

Wavelength
Ac-8

C32 H30 O6

510.56

296(2) K

$1.54178 \AA$ 
Crystal system

Space group

Unit cell dimensions

Volume

$\mathrm{Z}$

Density (calculated)

Absorption coefficient

$\mathrm{F}(000)$

Crystal size

Theta range for data collection

Index ranges

Reflections collected

Independent reflections

Completeness to theta $=68.20^{\circ}$

Absorption correction

Max. and min. transmission

Refinement method

Data / restraints / parameters

Goodness-of-fit on $\mathrm{F}^{2}$

Final R indices [I $>2 \operatorname{sigma(I)]~}$

$\mathrm{R}$ indices (all data)

Largest diff. peak and hole
Orthorhombic

P2(1)2(1)2(1)

$$
\begin{array}{ll}
\mathrm{a}=8.8004(3) \AA & \alpha=90.00^{\circ} . \\
\mathrm{b}=12.0028(4) \AA & \beta=90.00^{\circ} . \\
\mathrm{c}=26.0589(8) \AA & \gamma=90.00^{\circ} .
\end{array}
$$

2752.59(16) $\AA^{3}$

4

$1.232 \mathrm{Mg} / \mathrm{m}^{3}$

$0.686 \mathrm{mu}$

1080

$0.38 \times 0.26 \times 0.16 \mathrm{~mm}^{3}$

5.31 to $68.20^{\circ}$.

$-8<=\mathrm{h}<=10,-14<=\mathrm{k}<=14,-31<=\mathrm{l}<=28$

4602

$3453[\mathrm{R}$ (int) $=0.0300]$

$96.8 \%$

multi-scan

0.8982 and 0.7806

Full-matrix least-squares on $\mathrm{F}^{2}$

4602 / 0 / 346

1.281

$\mathrm{R} 1=0.0323, \mathrm{wR} 2=0.0823$

$\mathrm{R} 1=0.0624, \mathrm{wR} 2=0.1160$

0.177 and -0.171 e. $\AA^{-3}$ 
Compound 8 ( $\mathrm{R}=2$-biphenyl). Deposited in Cambridge Crystallographic Data Centre (CCDC\#268188)

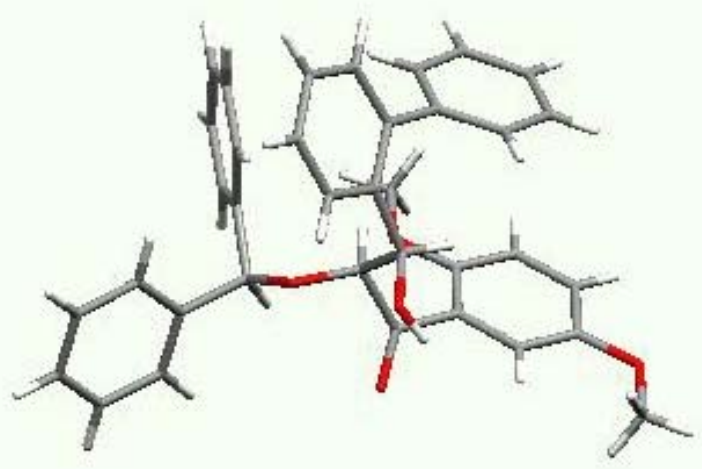

Table 3-S. Crystal data and structure refinement for 8 (R=2-biphenyl).

Identification code

Empirical formula

Formula weight

Temperature

Wavelength

Crystal system

Space group

Unit cell dimensions

Volume

$\mathrm{Z}$

Density (calculated)

Absorption coefficient

$\mathrm{F}(000)$

Crystal size

Theta range for data collection

Index ranges

Reflections collected

Independent reflections
8 (R=biphenyl)

C36 H32 O5

544.62

296(2) K

$1.54178 \AA$

Triclinic

P-1

$\mathrm{a}=9.8452(2) \AA$

$\alpha=110.479(10)^{\circ}$.

b 10.5613(3) A

$\beta=93.757(11)^{\circ}$.

$c=14.8694(5) \AA$

$\gamma=104.671(12)^{\circ}$.

\section{2}

$1.254 \mathrm{Mg} / \mathrm{m}^{3}$

$0.662 \mathrm{mu}$

576

$0.36 \times 0.36 \times 0.29 \mathrm{~mm}^{3}$

4.67 to $67.72^{\circ}$.

$-11<=\mathrm{h}<=11,-12<=\mathrm{k}<=12,-17<=\mathrm{l}<=16$

4541

$3641[\mathrm{R}(\mathrm{int})=0.0300]$ 
Completeness to theta $=67.72^{\circ}$

Absorption correction

Max. and min. transmission

Refinement method

Data / restraints / parameters

Goodness-of-fit on $\mathrm{F}^{2}$

Final R indices [I $>2$ sigma(I)]

$\mathrm{R}$ indices (all data)

Largest diff. peak and hole
$87.2 \%$

multi-scan

0.8311 and 0.7964

Full-matrix least-squares on $\mathrm{F}^{2}$

4541 / 0 / 373

1.054

$\mathrm{R} 1=0.0696, \mathrm{wR} 2=0.1838$

$\mathrm{R} 1=0.0816, \mathrm{wR} 2=0.1961$

0.342 and -0.248 e. $\AA^{-3}$

Compound 8 ( $\mathrm{R}=2$-furyl). Deposited in Cambridge Crystallographic Data Centre (CCDC\#268189)

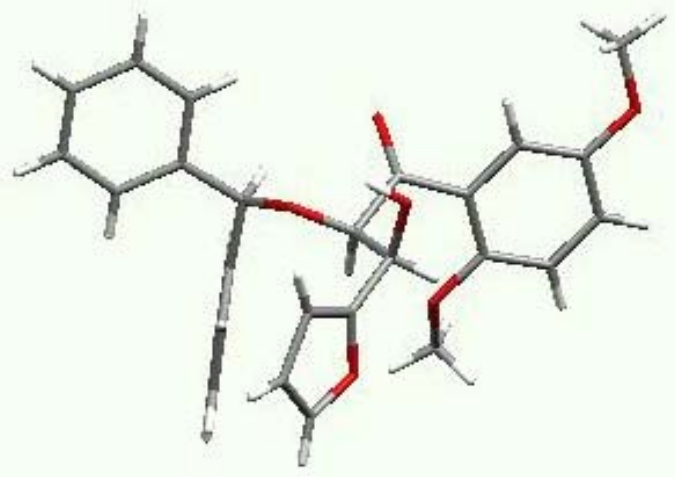

Table 4-S. Crystal data and structure refinement for $\mathbf{8}$ ( $\mathrm{R}=2$-furyl).

Identification code

Empirical formula

Formula weight

Temperature

Wavelength

Crystal system

Space group

Unit cell dimensions
8 (R=2-furyl)

C28 H26 O6

458.49

296(2) K

$1.54178 \AA$

Monoclinic

$\mathrm{P} 2(1) / \mathrm{C}$

$\mathrm{a}=12.2933(4) \AA$ $\alpha=90.00^{\circ}$. 
Volume

$\mathrm{Z}$

Density (calculated)

Absorption coefficient

$\mathrm{F}(000)$

Crystal size

Theta range for data collection

Index ranges

Reflections collected

Independent reflections

Completeness to theta $=68.89^{\circ}$

Absorption correction

Max. and min. transmission

Refinement method

Data / restraints / parameters

Goodness-of-fit on $\mathrm{F}^{2}$

Final R indices [I $>2 \operatorname{sigma(I)]~}$

$\mathrm{R}$ indices (all data)

Largest diff. peak and hole $\mathrm{b}=12.9966(4) \AA$

$\beta=109.518(2)^{\circ}$.

$\mathrm{c}=15.9109(6) \AA$

$\gamma=90.00^{\circ}$.

2396.02(14) $\AA^{3}$

4

$1.271 \mathrm{Mg} / \mathrm{m}^{3}$

$0.728 \mathrm{mu}$

968

$0.30 \times 0.26 \times 0.18 \mathrm{~mm}^{3}$

3.97 to $68.89^{\circ}$.

$-14<=\mathrm{h}<=14,-15<=\mathrm{k}<=15,-19<=\mathrm{l}<=18$

4260

$3971[\mathrm{R}(\mathrm{int})=0.0300]$

$98.5 \%$

multi-scan

0.8802 and 0.8113

Full-matrix least-squares on $\mathrm{F}^{2}$

4260 / 0 / 310

1.063

$\mathrm{R} 1=0.0410, \mathrm{wR} 2=0.1198$

$\mathrm{R} 1=0.0454, \mathrm{wR} 2=0.1225$

0.197 and -0.262 e. $\AA^{-3}$ 